\title{
Rassegna bibliografica sul volontariato italiano (1991-2010)
}

\author{
di Luca Corchia*
}

Il volontariato è un fenomeno emergente che, a partire dagli anni '70, è fuoriuscito dai ristretti ambiti della cultura e delle istituzioni caritatevoli e assistenziali, seguendo le complesse linee di differenziazione e integrazione della società italiana. Il punto di vista che proponiamo al fine di comprenderne le trasformazioni è totalmente interno alle scienze sociali: in conclusione del numero monografico della $\mathrm{Ri}$ vista abbiamo ritenuto opportuno di ripercorrere la storia delle rilevazioni, analisi e riflessioni che, dall'approvazione della legge-quadro 266/1991, hanno condotto la disciplina ad ampliare e diversificare le indagini e a focalizzare l'attenzione verso alcune fondamentali linee di tendenza, seguendo così la dinamica evolutiva del proprio oggetto di studio. E per quanto vi sia ancora un ritardo nell'esame di taluni mutamenti organizzativi, dietro cui sembra nascondersi una sorta di "snaturamento" dell'identità del volontariato, il bilancio sulla quantità e qualità degli studi degli ultimi tre decenni è certamente positivo.

Sino agli anni '80, il volontariato era un fenomeno ancora osteggiato dalle organizzazioni politico-ammministrative ed economiche della società italiana e un tema di ricerca del tutto residuale nelle scienze sociali e nell'opinione pubblica ufficiale. Il conflitto tra le rappresentanze del capitale e del lavoro e tra i grandi partiti di massa impediva di rivolgere lo sguardo verso le micro-rivoluzioni che nel mondo associativo stavano spostando il terreno delle rivendicazioni sulla "grammatica delle forme di vita": il contrasto delle nuove povertà ed emarginazioni, il riconoscimento di nuovi diritti per le donne, gli omosessuali, i carcerati, i malati, le minoranze etniche e religiose, l'interesse verso modelli eco-sostenibili, i temi della pace e della cooperazione sono tutti fenomeni culturali che circolano nella "microsfera della comunicazione quotidiana", si sedimentano in ambiti particolari e autonomi della sfera privata e trovano un fattore di aggregazione nelle esperienze del volontariato.

* Docente a contratto di Sociologia della comunicazione al Dipartimento di Scienze Politiche e Sociali dell'Università di Pisa, presso cui collabora per attività di ricerca e di progettazione territoriale. 
Durante gli anni '80, sono pochi gli intellettuali e gli accademici che ne colgono la portata. Di conseguenza, la bibliografia sul volontariato conservava una natura, per un verso, del tutto funzionale alle esigenze conoscitive e programmatiche delle realtà in cui le associazioni operano, per altro verso, di testimonianza documentale delle attività realizzate, in particolare dall'associazionismo cattolico, nel campo dell'assistenza ai "bisognosi". Il carattere territoriale caratterizza sia la copiosa documentazione finalizzata alla formazione dei volontari che le rilevazioni empiriche del fenomeno associativo, sempre più frequenti in conseguenza della diffusione di normative regionali e locali. Ne sono un esempio, quelle condotte in Umbria (1982), Veneto (1983), Piemonte (1986), Basilicata (1988), Trento (1985), Pavia (1986), Padova (1990), Verona (1986), Reggio Emilia (1987), Modena (1987), Milano (1985), Sanremo (1990), etc.. Alla frammentarietà territoriale delle indagini sul volontariato si aggiunge quella settoriale. Sul piano nazionale, infatti, l'interesse si limita ad aspetti molto specifici, come accade ad esempio nei documenti ministeriali sull'azione svolta dalle OdV nella lotta alla tossicodipendenza (1983) o sulla protezione civile $(1982,1983,1984)$ della Fondazione Emanuela Zancan. Per lo più, la letteratura sul volontariato continua a rimanere una espressione interna del mondo associativo, in particolare di ispirazione cristiana: sono numerose le pubblicazioni della Caritas Italiana su progetti e convegni sui temi del ruolo del volontariato cattolico nella "promozione umana sul terreno dell'assistenza e della prevenzione sociale" (1977), di "condivisione e liberazione" (1978) delle sofferenze e degli stati di bisogno degli emarginati (1977), dei poveri (1985), e sull'"ispirazione cristiana" $(1979,1984)$ del volontariato come momento costitutivo della "comunità cristiana" (1986) e "scelta di vita" (1987) dell'operatore credente.

Nell'uno e nell'altro caso, nel panorama italiano degli anni '80 sono prevalenti documenti ancora privi di una prospettiva interpretativa prettamente sociologica. Fanno eccezione, oltre al contributo di Bernardo Cattarinussi su Le associazioni volontarie nella riflessione sociologica occidentale e nella ricerca sociale italiana (1983), le ricerche che maturano intorno al Centro nazionale per il volontariato, costituito nel 1984 su iniziativa di istituzioni pubbliche e realtà associative ed ispirato dalla riflessione di Achille Ardigò sulla "Crisi di governabilità e mondi vitali (1980). In quell'anno, infatti, si svolse a Viareggio il Primo convegno nazionale "Volontariato e poteri pubblici", i cui atti furono pubblicati dalla rivista "Animazione sociale". Oltre a dar conto delle indagini svolte in precedenza dalla Fondazione G. Agnelli, il numero monografico comprendeva gli interventi di Ardigò, Bicocchi, Cesareo, Contessa, Labor, Lipari, Martini, Merlini, Nervo, Pacini, Rognoni, Scalia, Tavazza e Villasanta. Il Convegno nazionale diverrà un appuntamento a cadenza biennale in cui si incontreranno tutti i principali studiosi che definiscono il quadro interpretativo del volontariato italiano. Nel 1982 la rivista "Autonomie locali e servizi sociali" proponeva un intervento di ampia portata sul Volontariato, Welfare State e terza dimensione e nello stesso anno Achille Ardigò pubblicò in un rilevante volume a cura di Gianni Statera l'articolo L'approccio d'integrazione sistemica e $i$ suoi limiti. Comunicazione simbolica e "terza dimensione" in cui l'attenzione teoretica al "mondo della vita" si accompagnava alla fondazione di una 
sociologia della persona al centro di relazioni umane solidaristiche. Questa è la matrice principale degli studi sociali sul fenomeno del volontariato. Anche il decennio successivo si apre con il numero monografico della rivista "La Ricerca Sociale", a cura di Achille Ardigò e Ivo Colozzi, in cui viene posto il problema della conoscenza sociologica del volontariato, si compiono dei "bilanci" provvisori e si tracciano le "prospettive" che devono orientare gli studi della disciplina. Al volume parteciparono Laura Balbo, Francesco Belletti, Lucia Boccacin, Donatella Bramanti, Massimo Campedelli, Bernardo Cattarinussi, Ugo De Ambrogio, Pierpaolo Donati, Stefano Lepri, Costanzo Ranci, Giovanna Rossi, Alberto Tarozzi. Va riconosciuto che i loro contributi riuscirono a cogliere i temi che tutt'ora costituiscono alcuni dei maggiori ambiti di interesse degli studi sul volontariato, quali ad esempio la definizione del volontariato, la sua differenziazione rispetto ad altre forme di privato sociale, di mutuo aiuto, self help, cooperazione di solidarietà sociale, etc., la regolamentazione giuridica delle associazioni e le sue conseguenze, la natura del loro rapporto con gli enti pubblici e le imprese private, l'organizzazione dell'azione volontaria, gli ambiti di intervento e i destinatari, i riferimenti valoriali e motivazionali dei volontari. La "percezione" di essere di fronte a un "passaggio di fase" che impronta tutti i saggi, con la fine della "grande espansione spontanea" e l'inizio di una graduale istituzionalizzazione del fenomeno associativo, viene suffragata dall'approvazione della 1. 266/1991.

L'intento del presente articolo è quello di indicare i principali studi sul fenomeno del volontariato italiano, a partire dal riordino che la normativa ha imposto alle $\mathrm{OdV}$, nel quadro delle più generali trasformazioni sociali, economiche e culturali del nostro paese. Gli scritti sono suddivisi in alcuni nuclei tematici ricorrenti nella disciplina in Italia e che il numero della rivista "Sociologia e ricerca sociale" curato da Andrea Salvini intende riproporre e discutere, anche alla luce della letteratura internazionale.

Un primo insieme raccoglie quelli a carattere prevalentemente divulgativo sul volontariato, non profit e Terzo settore, che offrono delle rapide prospettive storiografiche e un quadro analitico sull'intero fenomeno: Rocchi (2003), Tomai (1994), Donati (1996, 1998), Degiacomi, Gilliavod (1996), Gawronski (1997), Gaeta, Prisciandaro (1998), Iovene, Viezzoli, Ascoli (1999), Comolli (2001), Barbetta, Maggio (2002), Barbetta, Cima, Zamaro (2003), Cartocci, Maconi (2006), Ranci (2006) e Licursi (2010).

Vi sono poi le indagini empiriche a carattere prevalentemente statistico o documentario il cui obiettivo è quello di fornire una descrizione delle organizzazioni di volontariato attraverso metodologie standard di tipo campionario o qualitative su casi di studio o strategie di ricerca mixed methods. All'interno di questo secondo insieme, possiamo suddividere ulteriormente gli studi assumendo come criterio l'ambito territoriale. In primo luogo, vi sono indagini - poche, purtroppo - concepite in un'ottica comparativa a livello europeo (Martinelli, 1991; Milanesi, 1993; Ardigò, 1994; Ascoli, Pavolini, 1999; Mancini, 2003; Caselli, 2009), comunitario (Ca- 
riello, 2003; Pellegrino, 2003) o inter-statuale (SPES, 2006). Altre ricerche, più numerose, sono condotte su di un ambito nazionale, soprattutto grazie all'iniziativa di istituzioni di settore, quali la Fondazione Italiana per il Volontariato (Frisanco, 1997, 2001, 2004, 2006; Ranci, Ascoli, 1997; Frisanco, Ranci, 1999; Poli, 2002), l'Osservatorio Nazionale del Volontariato (2001, 2006), l'European Volunteer Centre (2006) e l'Osservatorio Isfol (Turchini, 2006). Tra le ricerche nazionali occorre includere anche le sezioni sul volontariato e il Terzo settore contenute nelle molteplici indagini multiscopo del Censis su La situazione sociale del paese e dell'Istat sugli Aspetti della vita quotidiana. Dal 1998, l'Istituto Nazionale di Statistica pubblica dei rapporti su Le organizzazioni di volontariato in Italia e Le istituzioni non profit in Italia $(1998,1999,2000,2001,2005,2006,2008)$. Sull'attività dell'Istat si vedano anche gli scritti di Moreschi (2002), Moreschi e Brunetti (2001) e Moreschi e Zamaro (2001). Sono quasi del tutto assenti le indagini sul volontariato su scala macroregionale che raccolgano la tradizione degli studi sulle "Tre Italie" (Bagnasco, 1977) e le sub-culture territoriali (Cacagli, 1988; Catanzaro, 1989; Ramella, 2005; Baccetti, Messina, 2009). Si segnalano solo quelle sul Mezzogiorno dei primi anni '90 (Aa.Vv., 1991; Formez, 1991) e il Ciclo di seminari, tenutosi dal settembre 2007 al gennaio 2008, presso il Csv Aurora di Crotone (CESIAV, CSV Aurora, 2009). Ciò sorprende se si ricorda l'attenzione prestata al volontariato dell'Italia meridionale negli anni '80 da parte del Mo.V.I. (1985, 1989), del Formez (1986, 1990) e di due monografie curate da Cesareo e G. Rossi per le Edizioni Dehoniane $(1986,1989)$. Più numerosi, invece, sono i rapporti di indagine regionali, tra cui si evidenziano quelli realizzati nell'ambito delle attività di ricerca della Fondazione Italiana del Volontariato e del Centro Servizi Volontariato Toscana. In generale, occorre porre in rilievo il carattere fortemente frammentario e localizzato delle indagini empiriche sul volontariato, che quasi sempre sono il prodotto della collaborazione tra i centri o i gruppi di ricerca e la rete locale dei Centri di Servizio per il Volontariato, istituiti per lo più a livello provinciale, oppure l'esito scientificoeditoriale di rapporti occasionali con specifiche associazioni o con i settori delle politiche sociali delle amministrazioni comunali e provinciali.

La promulgazione della legge n. 266 dell'11 agosto 1991 rappresenta una cesura cruciale nella storia del volontariato italiano e in generale dell'ordinamento dello stato sociale. La legge-quadro è il punto di arrivo di una lunga discussione, avviata nel 1980 dal senatore Nicolò Lipari al primo Convegno nazionale del Volontariato di Viareggio ed affidata all'attività parlamentare con la proposta di legge n. 575 del 13.3.1984. La sua approvazione, non poco contrastata, e quella dei decreti attuativi sono l'esito di una vicenda non solo giuridica complessa che coinvolge numerosi attori della politica e del mondo associativo organizzato: molteplici sono gli orientamenti culturali, gli interessi più contingenti e le sensibilità che il Legislatore ha dovuto mediare nel tentativo di riordinare una normativa disomogenea che rischiava di assumere le sembianze di un "vestito di Arlecchino", con i conseguenti squilibri tra le Regioni. Sin dal convegno organizzato dal Centro Nazionale per il Volontariato nel dicembre del 1991, i problemi normativi e i loro risvolti fattuali sono stati 
posti al centro della riflessione scientifico-accademica e del confronto nella sfera pubblica politica. Non sorprende, dunque, che una parte significativa della letteratura riguardi la legge-quadro e i decreti attuativi, l'evoluzione della disciplina e le proposte di modifica (Bassi, 1992; Rei, 1992, 1994; Bruscuglia, 1993; Mariconda, 1993; Manganozzi, 1993, 2005; Boccacin, 1994; Sciumè, 1996; Springhetti, 1996; E. Rossi, 1997, 2009; Lipari, 1998; Rao 1999), le normative regionali e gli ordinamenti locali (Anci-Upi, Centro Nazionale Volontariato, Fondazione E. Zancan, 1991; Corghi, 1991; Aa.Vv., 1992; Bassi, 1993, 1994; Donatelli, 1998; Rei, 1999; Dal Canto, 2009; Costa, 2009), e alcuni aspetti civilistici, contabili e fiscali (Colombo, 1993; Pinto, 1996; Tavazza, 1998; Angeloni, 1999; Ragghianti, 2000, 2002, 2004, 2005; Saccone, Spagnoletti, 2001; E. Rossi, 2010).

Un quarto insieme di pubblicazioni, molto diversificato al suo interno, raccoglie degli interventi che si potrebbero definire identitari e di prospettiva, nel senso che tematizzano il problema della natura del volontariato all'interno del campo più ampio del Terzo settore, ne discutono le linee di tendenza ed esprimono giudizi valutativi.

Per un verso, alcuni studi propongono un approccio unificato al Terzo settore che comprende tutte le organizzazioni mutualistiche e senza fini di lucro (Gui, 1991; Bassi, 1994) mentre altri approfondiscono la specificità culturale del volontariato (Colozzi, 1994; Cecconi, 1999; Boccacin, Bramanti, 2000; G. Rossi, Boccacin, 2004, 2006; Napo, 2007; Nervo, 2008; Longhi, 2009). Il problema dei rapporti fra istituzioni non profit e associazioni di volontariato - al centro del Convegno organizzato nel Comune di Fiè allo Sciliar in Provincia di Bolzano tra il 15 e il 19 maggio 1995 dalla Fondazione E. Zancan, a cura di Bonini et al. - è stato esaminato negli scritti di Bramanti (1996), Ranci (1996), Ranci Ortigosa (1996), Boccaccin (1992, 1997), Borzaga (1997, 1998, 2000), Fiorentini (1997), Belletti (1999), La Rosa (2001), Boccacin, G. Rossi, (2003), G. Rossi, Colozzi (2004), Boccacin (2005), Donati (2004), Martello (2009). Per altro verso, si è affermata una tradizione di ricerca sociologica attenta allo sviluppo e alle trasformazioni delle organizzazioni di volontariato nel quadro dei fenomeni della cosiddetta modernità avanzata. Tra gli studiosi vi sono Garelli (1992), Campedelli et al. (1996), Comolli, Garbagnati (1996), Donati (1996), Vittadini (1997), Dal Dosso (1998), Tavazza (1998), Martini (1999), Barbetta (2000), Borzaga, Fazzi (2000), Fazzi (2001, 2002), Paltrinieri (2001), Nervo (2002), Antonelli, Nosvelli (2003), Ascoli (2003), Donati, Colozzi (2004), Ambrosini (2005), Frisanco (2006, 2009) e Salvini (2009, 2010).

È certo un merito della sociologia italiana quello di non avere ricondotto la crescita del volontariato soltanto alla crisi di un welfare state alle prese con i vincoli di spesa pubblica e sempre inadeguato di fronte alla differenziazione dei bisogni degli utenti e alle aspettative di autonomia locali e partecipazione dei cittadini. Al di là della funzione di servizio svolta dal volontariato in molteplici campi delle politiche sociali, socio-sanitarie, culturali, di tutela e promozione dei diritti e cooperazione internazionale, di protezione civile e ambientale, viene sottolineata la sua importanza come "fatto associativo" caratterizzato da un modello relazionale che "incorpora" i valori dell'altruismo, della solidarietà, della reciprocità e della promozione 
umana e sociale. Tra i contributi più pertinenti vi sono i volumi a cura di Borzaga (1991) e Franzoni (1993) per la Fondazione E. Zancan, quelli di Ranci, De Ambrogio e Pasquinelli (1991), Ascoli e Pasquinelli (1993), Colozzi (1994), Cotesta e Bartocci (1995), Fadda (1996), G. Rossi (1997), De Leonardis (1998), Stanzani (1998), Vittadini (1998, 2003), Ascoli (1999), Fazzi e Messora (1999), Nervo (1999), Ranci (1999), Prezioso (2000), Cattaneo (2001), Boccacin (2003), Pavolini (2003), Accorini (2008), il numero monografico degli "Studi Zancan: Politiche e Servizi alle Persone» su Il terzo settore nei sistemi locali di welfare (2007) e i saggi di Boccacin (1994), Ranci (1994), Natale (1994), Ambrosini (1995), Donati (1996, 2006), Colozzi (1998, 2000), Bassi (2000), Costa (2002), Frisanco (2005), Pizzolato (2005), Licursi, Marcello (2010) e Salvini (2010).

Un numero considerevole di scritti tematizza il ruolo del volontariato organizzato quale fattore di accrescimento del livello di coesione sociale, spirito civico, partecipazione democratica e di promozione e tutela dei diritti sociali, quale precondizione di esercizio di quelli civili e politici. Tra i molti contribuiti si segnalano quelli del Censis (1991), Tomai et al. (1991), P. De Nardis (1993), Donati (1993, 1997, 2007), Vecchiato, Pasini et al. (1995), Boccaccin (1996, 2006), Saccone (1996), Colozzi (1997), Cotturri (1998), Mutti (1998), Nervo (1999), Pasini G. et al. (1999), Diani (2000), IREF (2000), Donati, Colozzi (2001, 2002, 2006), Barbetta (2002), Caltabiano (2003), La Valle (2003), Arcidiacono (2004), Cesareo (2004), De Luca (2004), Moro (2005), Nervo (2004), Salvati (2004), G. Rossi, Cartocci (2007), Bartholini (2008), Giusti, Caldelli (2008), Boccaccin (2009), Guidi (2009) e Cordaz (2010).

Un'ulteriore dimensione del volontariato che è stata esaminata negli studi sociologici è il radicamento dei valori, delle solidarietà e delle motivazioni dei volontari nel fertile humus dei legami familiari e delle relazioni amicali e di vicinato proprie dei gruppi primari. A tale riguardo, i principali contributi sono quelli di De Palma (1998), G. Rossi (2003, 2004), Marta (2004), Marta, Pozzi (2004), Donati, Colozzi (2006). Parallelamente, un'altra agenzia di socializzazione che costituisce benefici rapporti di contaminazione con il volontariato è la scuola primaria e secondaria. Tra i numerosi scritti menzioniamo solamente quelli più recenti di Maccarini (2006), Buzzi (2007), Guglielmi, Buzzi (2007), Mattei (2007), Fradeani (2008) e Siringo (2010).

Proprio in ragione della specifica identità del volontariato, un campo di ricerca rilevante riguarda la possibile "colonizzazione" di questa realtà emergente del mondo della vita da parte delle logiche delle organizzazioni politico-amministrative ed economiche. Rispetto al sistema pubblico, gli strumenti di raccordo tra le associazioni di volontariato e gli enti locali stanno generando un fenomeno di "isomorfismo organizzativo" ed etero-direzione di fini e condotte (Gualandi E. et al., 1992; Leone, 1998; Anfossi, 1999; Dalla Mura, 1999, 2003; Migliorini, 1999; Castegnaro, 2002; Bezzi, 2005 Minetti, 2005; Buizza, 2006, Andreani, Minà, 2007; Salvini, 2007; Cangelosi, 2010). Rispetto al sistema economico, il tema delle relazioni tra le OdV e le imprese, e quindi tra la solidarietà e il profitto, e l'apporto del volontariato allo sviluppo del paese sono l'oggetto di attenzione di molti studiosi tra i quali 
Franzoni (1993), Boccacin Lucia (1994), Mari (1994), Barbetta (1996), Alessandra Merlo (1996), Zamagni (1996, 1998, 2002), D’Orazio (1998), Tubaro (1999), Musella, D'Acunto (2000), Piga (2000), Archibugi (2002), E. Rossi (2004), Bruni (2007) e Garelli, Guerra (2009). Dalle ricerche sembra emergere, certo, l'importanza della realtà associativa nella riproduzione materiale della società ma anche un mutamento che coinvolge la "mission" dell'azione volontaria, con il passaggio da un approccio "vocazionale" a uno "utilitarista". In particolare, un aspetto che ha suscitato particolare interesse è quello dell'occupazione retribuita dei volontari nelle imprese sociali e cooperative sociali: (Lunaria, 1997), Orioli (1997), IREF (1998), Borzaga (2002, 2003), Frisanco (2002), Crescenzi, Bonacini (2005) e Fiorillo (2008).

L'approssimarsi delle organizzazioni di volontariato a modelli propri della pubblica amministrazione e delle imprese private è strettamente connesso alla funzione integrativa e sostituiva che esse svolgono in numerosi ambiti della realtà sociale, culturale e ambientale del paese. Di conseguenza, risulta sempre più cospicua la letteratura sugli interventi di servizio erogati dalle OdV a favore della collettività o di particolari gruppi. Nella presente bibliografia ci limitiamo a segnalare gli studi che rivolgono il proprio interesse al ruolo del volontariato nelle politiche sociali e sociosanitarie dopo la crisi del welfare state, in particolare nella gestione e nella promozione di buone pratiche nei servizi alla persona, nella lotta all'esclusione sociale, nell'integrazione degli immigrati, nel campo della giustizia e nel "trattamento" dei detenuti nelle carceri, etc. A tale riguardo, si possono utilmente consultare gli scritti di G. Rossi (1993, 1994, 1997), Fadda (1993), Dolazza L. (1994), Fazzi (1994, 2002), Folgheraiter (1994), Sgritta (1994), Boccacin (1995), Borzaga, Gui, Schenkel (1995), Coppola (1996), Marzotto (1995, 1996), Piazza (1995), Colozzi (1997), Lovati (1997), De Siervo (1998), Gastaldi, Springhetti (1998), Anoni, Borselli (1999), Ascoli, Pavolini (1999), Caritas Italiana (1999), Caselli, Galli, Settesoldi (1999), Marsico (1999), Consulta Nazionale volontariato in Sanità (2000), Donati (2000), Fiorentini (2000), Frisanco (2000), Nervo (2001), Bonomi, Portioli (2003), Delai (2003), Cesvot (2004), D`Angelo, Gallo, Santanera (2005), Rovati (2005), Mosca (2008), E. Rossi (2009), G. Rossi, Boccacin (2009), Ferrari (2007), Gaudio, Caramelli (2009) e Monetini (2009).

Un insieme di studi riguarda il volontariato in quanto fenomeno organizzativo, che richiede nuove capacità di gestione, promozione, controllo della struttura, la formazione dei volontari, la comunicazione interna ed esterna e la ricerca delle risorse.

La prima questione concerne la sfida di coniugare la solidarietà e l'efficienza nella direzione, promozione e valutazione delle organizzazioni non profit. A partire dagli anni ' 90 , concetti come management e marketing e prospettive provenienti dall'economia aziendale hanno fatto il loro ingresso nel lessico dei volontari e degli studiosi del volontariato, accentuando - a dispetto delle sue peculiarità organizzative - il rilievo della leadership nelle OdV, la tendenza alla loro professionalizzazione dei responsabili, una gestione del personale e una rendicontazione delle attività sempre più simile a quella delle imprese. Gli scritti di riferimento sono quelli di Ambrosini (1992, 1994, 1999, 2000), Fiorentini (1992, 1996), Cavenago (1996), 
Colozzi (1996), Spreti (1996), Fossati (1997), Agenzia per lo sviluppo del non profit (1998, 2002), Ambrosio, Bandini (1998), Busnelli, Gioia (1998), Busnelli, Giuliani (1998), Milanese (1998), Morganti (1998), Cominelli (1999), Mason, Melandri (1999), Matacena (1999), Ambrosio, Bonacina (2000), Barbetta, Schena (2000), Cerri (2000), Fazzi (2000, 2001), Morganti $\left(2000,2010^{2}\right)$, Crescenzi $(2002,2003)$, Atzei (2003), Converso, Piccardo (2003), Lertora (2003), Sibilio Parri (2003), Tronca (2003), Catalano (2004), Bagnoli (2005, 2007) Hinna (2005), Ragghianti, Settesoldi (2007), Colozzi, Prandini (2008), Bilotti, Nasi, Tola, Volterrani (2009), Fondazione E. Zancan (2009) e Macchioni (2010).

Un secondo tema che contraddistingue la riflessione sull'organizzazione delle OdV riguarda la formazione dei volontari: il "sapere", il "saper fare" e il "saper essere". A tale proposito si vedano gli studi di Bocca (1993), Bramanti (1993, 1994, 1996), Rocchi (1993), Prenna (1995), Bellamio (1996), Beretta, Melandri (1996), Busnelli (1996), Gangeri (1996), Melandri (1996, 1997), De Palma (2002), Converso, Piccardo (2003), Pellegrini (2005), Rizza (2005), Sapienza (2005), Tomassini (2005), Sordelli (2010).

Risulta, per contro, poco approfondito il problema della comunicazione interna alle OdV e della comunicazione esterna verso i pubblici di riferimento. Fanno eccezione le ricerche della FIVol - Volontariato e informazione, a cura di Franzoni (1993) e La voce del volontariato, a cura di Frisanco, Trasatti, Volterrani (2000) -, il volume a cura di Tisselli (1999), con contributi di Albanesi, Bonacina, Franzoni, Martini, Mori e Volterrani, gli scritti di Kanzian (1999), Volterrani (1994, 1999), il libro di Pira (2005), l'indagine sulla La comunicazione del Terzo Settore nel Mezzogiorno, a cura di Martelli (2006), quella di Springhetti (2008) e i saggi di Martelli (2008), Gili (2008) e Sobrero (2008). Altrettanto trascurato è l'ambito di studio sulla "propensione al networking" da parte delle OdV. L'orientamento favorevole alle collaborazioni è un fattore importante dal punto di vista della capacità organizzativa, dello scambio di esperienze e risorse, dell'accesso a risorse non altrimenti disponibili, e della più generale predisposizione all'apertura. Al di là degli aspetti strategici del networking, infatti, ciò che interessa è che il "far rete", in primo luogo all'interno del mondo del volontariato, può essere assunto come indicatore di "coesione sociale" e costruzione di "capitale sociale". La pratica della collaborazione promuove quello "spirito della reticolarità" che consolida un atteggiamento orientato al possibile sviluppo della solidarietà. Tra pochi contributi allo studio delle partnership vi sono quelli di Ambrosini, De Bernardis (1999), Gigni (2003), Salvini, Cordaz (2007), Boccacin (2010) e Bramanti (2010).

Riscuote maggior interesse da parte degli studiosi (e dei Centri di servizio che li finanziano) il tema del fundraising e people raising, ovvero la ricerca delle risorse materiali e umane necessarie al mantenimento delle OdV. Ed è pur vero che senza l'autofinanziamento o il tesseramento da parte dei soci e dei volontari, le sovvenzioni statali o di enti locali o internazionali, gli sponsor, i lasciti, gli introiti derivanti da specifici eventi, la partecipazione a bandi e i premi in denaro, è impossibile generare solidarietà. A tale riguardo si vedano gli scritti di Cacopardo (1996), Castegnaro (1996), Manfredi (2001), Griffino (2002), Ellis, Melandri (2003), Bemi 
(2004, 2008), De Palma (2004), Lemmetti (2004, 2006), Gazzola (2005), Martello, Zicari (2009) e Solfrini (2010).

Molti studi si interessano ai profili dei volontari, indagandone la composizione socio-demografica a livello nazionale, macroregionale o locale, le motivazioni personali, le aspettative di comportamento e i riferimenti valoriali del volontario. Tra i tanti, Franzoni (1993), Milanesi (1993), Sarpellon (1993), Cattarinussi (1994), G. Rossi (1996), Degiacomi, Gilliavod (1997), IREF (1998), Capanna, Steca, Imbimbo (2002), Barbaranelli, Caprara, Capanna, Imbimbo (2003), Fedi, Greganti, Tartaglia (2004), Pieroni (2005), Marta, Guglielmetti, Pozzi (2005), Marta, Pozzi (2007), Previtali (2007), Spedicato Iengo (2007), Degli Antoni (2009), Psaroudakis (2010), Romano, Todaro (2010). Numerose ricerche, in particolare, approfondiscono la conoscenza del volontariato da parte dei giovani e la loro disposizione a tale esperienza. Tra questi si vedano almeno gli scritti di Franzoni (1993), Badolato, Rizzi (1994), D’Orazio (2001), Ivaldi, Polidori (2002), Marta, Scabini (2003), Ambrosini (2004), Boccacin (2004), Frisanco (2004, 2007), G. Rossi (2004), Marta (2004), Ambrosini (2005), Pirozzi (2006), Guizzardi Luca (2007), Di Gioia, Giacomello, Inserra, Rotondi (2010) e Salvini (2010).

Costituiscono un ambito di studio interessante ancora da approfondire le ricerche sulla rappresentazione pubblica del volontariato nei mezzi di comunicazione di massa, nella stampa, in televisione e nei nuovi media: Bonacina (1998), Balzanelli (1999), Fedi, Gattino (2004), Forestieri, Concetta (2004), Volterrani (2006) e Sorrentino (2007).

Un ultimo insieme di scritti riguardano le realtà organizzative di rappresentanza, coordinamento e confronto del volontariato italiano, quali la Fondazione Italiana per il Volontariato (Tavazza, 1999), il Movimento volontariato italiano (Carretta, 1996), il Centro Nazionale per il volontariato (Martini, 1996), i Forum del Terzo settore l'Università del Volontariato (Macaluso, 1996; Anconelli, Piccinini, 2005) e, soprattutto, i Centri di servizio per il volontariato (Memo, 1997; Pancaldi, 1998; Merlini, 1999; Collegamento nazionale centri di servizio per il volontariato, 2000, 2001, 2004; Granelli, 2001; E. Rossi, 2001; Martelli, 2009; Ampollini, 2000; CSVnet, 2010).

Prima di elencare gli scritti suddivisi per aree tematiche è opportuno precisare che, per ragioni di economia espositiva, abbiamo dovuto escludere dalla rassegna bibliografica quasi tutti gli interventi brevi, seppur rilevanti, pubblicati su "Animazione Sociale", "Impresa sociale", "Mondo sociale", "Prospettive Sociali e Sanitarie", "Servizi sociali oggi", "Terzo Settore", "Vita", o su riviste espressione diretta del mondo associativo, quali la "Rivista del Volontariato", mensile edito dalla Fondazione Italiana del Volontariato, "Volontariato Oggi", agenzia cartacea del Centro Nazionale per il Volontariato e "Fogli di Informazione e di Coordinamento", a cura della Federazione regionale Lombardia del Movimento di volontariato italiano. Ci limitiamo a segnalare che, nonostante i numerosi archivi, centri di documentazione e gruppi di ricerca sul volontariato, non è ancora disponibile una bibliografia completa. 


\section{Volumi divulgativi}

S. Rocchi (1993), La formazione dell'operatore volontario, in Id. (a cura di), Il volontariato fra tradizione e innovazione, Roma, La Nuova Italia Scientifica.

B. Tomai (1994), Il volontariato. Istruzioni per l'uso, Milano, Feltrinelli.

P. Donati (1996), Sociologia del terzo settore, Roma, La Nuova Italia Scientifica.

C. Degiacomi, M.R. Gilliavod (a cura di) (1996), Volontariato, Volontariati. Guida Ragionata, Torino, Edizioni Radionotizie.

G.P. Barbetta (1997), The Nonprofit Sector in Italy, Manchester, Manchester University Press.

S. Gawronski (1997), Guida al volontariato. Un libro per chi vuol cominciare, Torino, Einaudi.

P. Donati (a c. di) (1998), Sociologia del terzo settore, Roma, Carocci.

S. Gaeta, V. Prisciandaro (1998), I volti della solidarietà. Manuale pratico di volontariato, Torino, Edizioni San Paolo.

N. Iovene, M. Viezzoli, U. Ascoli (1999), Il libro del terzo settore: l'universo del non-profit tra impresa e solidarietà sociale, Roma, Adn Kronos Libri.

G.M. Comolli (2001), Volontariato perché sì e quando no. Manuale per il volontariato di ieri, di oggi e di domani, San Pietro in Cariano (VR), Il Segno Gabrielli Editori.

G.P. Barbetta, F. Maggio (2002, 2008²), Non profit, Bologna, il Mulino.

G.P. Barbetta, S. Cima, N. Zamaro (a c. di) (2003), Le istituzioni nonprofit in Italia. Dimensioni organizzative, economiche e sociali, Bologna, il Mulino.

C. Ranci (2006), Il volontariato: [i volti della solidarietà], Bologna, il Mulino.

R. Cartocci, F. Maconi (a c. di) (2006), Libro bianco sul Terzo settore, Bologna, il Mulino.

S. Licursi (2010), Sociologia della solidarietà, Roma, Carocci.

\section{Indagini statistiche}

\subsection{Raffronti a livello europeo}

T. Martinelli (a c. di) (1991), Il volontariato in Europa, Lucca, Centro nazionale per il volontariato.

Unione Italiana del Lavoro (a c. di) (1991), Associazionismo volontariato cooperazione sociale. Nuove solidarietà, nuovi bisogni in Italia e in Europa, Atti del convegno, Consiglio Regionale della Toscana, 10-11 ottobre 1991, Roma, Ancs-Uil.

G. Milanesi (1993) (a c. di), «Volontariati in Europa», Quaderni di Volontariato, 3, Roma, Fondazione italiana per il volontariato.

L. Boccacin (1993), «Il terzo settore in Germania e in Italia: elementi per una comparazione», in Studi di Sociologia, 3, pp. 269-282.

A. Ardigò (1994), Volontariato e welfare state in Europa: il difficile cammino verso e oltre lo stato del benessere, in I. Colozzi (a c. di), Terzo settore e nuove politiche sociali in Italia e in Europa, Milano, FrancoAngeli, poi in Rivista Servizi Sociali, 1, 1995, suppl.

U. Ascoli, E. Pavolini (1999), «Le organizzazioni di Terzo settore nelle politiche socioassistenziali in Europa: realtà diverse a confronto», Stato e Mercato, 57, pp. 441-476.

IREF-ACLI (1999), Senza frontiere. L'associazionismo in Europa, Roma, Editoriale AESSE.

T. Cariello (2003), «La Comunità europea e il Terzo settore», Non profit, IX, 3, pp. 557-587.

S. Mancini (2003), «Il volontariato e la cittadinanza attiva in Europa», La Scuola in Europa, III, 3, pp. 68-75.

Centro di Servizio per il Volontariato del Lazio (2006), Italia, Id., Il volontariato in Europa. 
Organizzazioni, promozione, partecipazione. Spagna, Francia, Regno Unito, Paesi Bassi, Polonia, Repubblica Ceca, Italia, Roma, Spes, pp. 241-275.

M. Caselli (2009), «Il volontariato in Italia e in Europa. Riflessioni in vista del 2011 Anno Europeo del Volontariato», Politiche Sociali e Servizi, XI, 1, pp. 41-52.

\subsection{Ricerche a livello nazionale}

I. Colozzi, A. Bassi (1995), Una solidarietà efficiente: il terzo settore e le organizzazioni di volontariato, Roma, NIS.

S. Accorso Caroli et al. (1996), Atlante del volontariato italiano, Roma, Cgil Auser.

L. Bobba, A. Nanni (1997), Viaggio nel Terzo settore. Parlano i protagonisti, Torino, Edizioni Sonda.

R. Frisanco (a c. di) (1997), Rilevazione FIVol 1997 sulle organizzazioni di volontariato sociale italiano. Primi Risultati, Roma, FIVol.

C. Ranci, U. Ascoli (a c. di) (1997), La solidarietà organizzata. Il volontariato organizzato oggi, Roma, FIVol.

V. Italia, A. Zucchetti (1998), Le organizzazioni di volontariato, Milano, Giuffrè.

F. Santanera, A. Gallo (1998), Volontariato. Trent'anni di esperienze: dalla solidarietà ai diritti, Torino, Utet.

R. Frisanco, C. Ranci (a c. di) (1998), Le dimensioni della solidarietà. Secondo rapporto sul volontariato sociale italiano, Roma, FIVol.

R. Frisanco, C. Ranci (a c. di) (1999), La solidarietà organizzata, Roma, FIVol.

Istat (1999), Le organizzazioni di volontariato in Italia. Strutture, risorse e attività, Argomenti, Roma.

Istat (2000), Le organizzazioni di volontariato in Italia. Anno 1997, Note rapide, Roma.

R. Frisanco (2001), Le organizzazioni di volontariato alla terza rilevazione Fivol 2001, Roma, FIvol.

Istat (2001), Le organizzazioni di volontariato in Italia. Anno 1999, Note rapide, Roma.

Istat, (2001), Istituzioni nonprofit in Italia. I risultati della prima rilevazione censuaria. Anno 1999, Roma.

Dipartimento per gli Affari Sociali, Osservatorio Nazionale del Volontariato (a c. di) (2001), Rapporto biennale sul volontariato in Italia 2000, Presidenza del Consiglio dei Ministri, Roma.

M. Brunetti, B. Moreschi (2001), «Le rilevazioni Istat sulle organizzazioni di volontariato», Autonomie locali e servizi sociali, 2, pp. 261-270.

B. Moreschi (2002), «Il primo censimento Istat delle istituzioni e delle imprese non profit: Una sintesi», Autonomie locali e servizi sociali, 1, pp. 101-112.

A. Poli (2002), Volontariamente, oggi: le organizzazioni di volontariato in Italia, Roma, FIVol.

R. Frisanco (2004), «Dati statistici: lettura e interpretazione del volontariato e del Terzo settore», Oggi Domani Anziani, 2, pp. 127-156.

Istat (2004), Le organizzazioni di volontariato in Italia. Anno 2001, Statistiche in breve, Roma.

Istat (2005), Le organizzazioni di volontariato in Italia. Anno 2001, Informazioni, Roma.

Istat (2005), Le organizzazioni di volontariato in Italia. Anno 2003, Statistiche in breve, Roma.

C. Caltabiano (2006), Altruisti senza divisa: storie di italiani impegnati nel volontariato informale, Roma, Carocci. 
R. Frisanco (2006), Volontariato sotto la lente: lo scenario del volontariato organizzato alla luce della quarta rilevazione FIVol 2006, Roma, FEO-FIVol.

Ministero del Lavoro e delle Politiche Sociali, Osservatorio Nazionale per il Volontariato (2006), Volontariato: Rapporto biennale sul volontariato in Italia 2005: (Statistical Data Available for 2003), Roma, Tipografia A.G.M. Martelli.

Ministero del Lavoro e delle Politiche Sociali, Osservatorio Nazionale per il Volontariato (a c. di) (2006), Sintesi del Rapporto biennale sul volontariato in Italia: 2005, Roma.

Istat (2007), Le organizzazioni di volontariato in Italia. Anno 2005, Statistiche in breve, Roma.

Cnel-Istat (2008), Le organizzazioni di volontariato, in Dimensioni e caratteristiche strutturali delle istituzioni nonprofit in Italia, Primo rapporto CNEL/ISTAT sull'economia sociale, Roma, pp. 34-58.

\subsection{Ricerche a livello macroregionale e regionale}

AA.VV. (1991), Il Volontariato nel Mezzogiorno, Acireale (Ct), Bonanno.

Formez (1991), «L'azione volontaria nel mezzogiorno», Autonomie locali e servizi sociali, XIV, 1, pp. 23 ss.

E. Campelli (1994), Solidarietà ed etica laica. Un'indagine sul volontariato in Emilia Romagna, Milano, FrancoAngeli.

V. Cesareo, G. Rossi (1994), Il volontariato in Lombardia, Vita e Pensiero, Milano.

Osservatorio meridionale, Regione Calabria (1995), Dove va il volontariato in Calabria? Materiale di documentazione, Osservatorio meridionale, Reggio Calabria.

M.E. Martini (1996), Il volontariato in Toscana, Giunta della Regione Toscana, Firenze, 3031.5.1996.

Bassi Andrea, Stanzani Sandro (1997), Il volontariato in Emilia Romagna, Roma, FIVol.

R. Serra, G. Giorio (1997), La sollecitudine per gli altri. Realtà e tendenze del volontariato in Friuli-Venezia Giulia, Gorizia, I.S.I.G..

R. Frisanco (1999), «Le organizzazioni di volontariato in Liguria», Prospettive Sociali e Sanitarie, $11, \mathrm{pp} .13-15$.

A. Salvini (1999), Identità e bisogni del volontariato in Toscana, n. 7, Firenze, Cesvot.

C. Caltabiano (2001), L'attivazione della solidarietà. Primo rapporto SIS sull'economia sociale in Sardegna, Milano, FrancoAngeli.

M. Pendenza (2001), Le organizzazioni di volontariato in Campania, Napoli, Liguori.

AA.VV. (2002), Il volontariato nelle Marche, anno 2002: mappa e caratteristiche strutturali ed evolutive del volontariato nelle Marche: guida alle associazioni di volontariato delle Marche, Ancona, Centro di Servizio per il Volontariato AVM.

C. Caltabiano (a c. di) (2002), Lombardia solidale. Terzo settore e civismo in una regione in transizione, Milano, FrancoAngeli.

Centro di servizio per il volontariato della Valle d'Aosta (2002), Le organizzazioni di volontariato in Valle d'Aosta, Aosta, Tipografia Valdostana.

M.L. Chiambretto (a c. di) (2002), Il volontariato in Piemonte: la realtà piemontese nella rilevazione FIVol 2001, Acqui Terme, Cooperativa sociale Impressioni Grafiche.

C. Crucitti (2003), Manuale del Volontariato. Volontariato in Calabria. Associazioni ed Organizzazioni, Catanzaro, Quaderni mediterranei.

R. Frisanco (a c. di) (2003), Il volontariato in Sicilia: [le organizzazioni di volontariato nella] rilevazione FIVOL 2001, Roma, Essegraf.

R. Frisanco (a c. di) (2003), Il volontariato nel Lazio: Rilevazione Fivol 2001, Roma, Essegraf. 
R. Frisanco (a c. di) (2003), Il volontariato nel Veneto: Rilevazione Fivol 2001, Carmignano di Brenta, Artegreafica Munari.

R. Frisanco, P. di Giammatteo (a c. di) (2003), Mappa e caratteristiche strutturali ed evolutive delle organizzazioni di volontariato in Umbria: rilevazione 2001, Perugia, Tip. Perugina.

R. Frisanco, S. Limberti (a c. di) (2003), Il volontariato in Toscana: Rilevazione Fivol 2001, Roma, Essegraf.

R. Frisanco, M. Pentassuglia (a c. di) (2003), Il volontariato in Friuli Venezia Giulia: rilevazione Fivol 2001, Roma, Essegraf.

R. Frisanco, S. Malena (a c. di) (2003), Il volontariato in Trentino: la rilevazione FIVOL 2001 e le associazioni iscritte nei pubblici registri, Trento, Giunta della Provincia autonoma.

R. Frisanco (a c. di) (2004), Il volontariato in Campania: Rilevazione Fivol 2001, Roma, Essegraf.

R. Frisanco (a c. di) (2004), Il volontariato in Puglia. Rilevazione Fivol 2001, Roma, Essegraf.

R. Frisanco, M. Vender (a c. di) (2004), Il volontariato in Basilicata. Rilevazione Fivol 2001, Roma, Essegraf.

R. Frisanco, M.C. Arabia (a c. di) (2004), Il volontariato in Calabria: Rilevazione Fivol 2001, Roma, Essegraf.

S. Menna (a c. di) (2004), Il volontariato in Lombardia: Rilevazione Fivol 2001, Roma, Essegraf.

A. Guglielmin (2004), L'Associazionismo in Friuli Venezia Giulia, Udine, IRES Friuli Venezia Giulia.

A. Amodeo, G. De Luzenberger (a c. di) (2005), In ascolto del volontariato. L'esperienza di un percorso di partecipazione, Napoli, Regione Campania.

A. Amodeo, G. De Luzenberger (2005), Identità e ruolo del volontariato organizzato nella costruzione del sistema di welfare locale in Campania, Napoli, Regione Campania.

A. Salvini, D. Cordaz (a c. di) (2005), Le trasformazioni del volontariato in Toscana. II rapporto di indagine, n. 27, Firenze, Cesvot.

M. Golinelli, G. Scidà (2006), Volontariato, appartenenza associativa, capitale sociale: un legame non scontato nella realtà romagnola, in P. Donati, I. Colozzi (a c. di), Terzo settore e valorizzazione del capitale sociale in Italia: luoghi e attori, Milano, FrancoAngeli, pp. 191-212.

E. Ranci Ortigosa (2006), Il terzo settore nel Mezzogiorno: Principali differenze rispetto al resto del Paese, Seminario, Napoli, 14.1.2006.

S. Stanzani (2006), Le differenze del Mezzogiorno. Il profilo delle regioni interessate dalle indagini sul terzo settore secondo l'Istat, in S. Martelli (a c. di), La comunicazione del Terzo Settore nel Mezzogiorno, Milano, FrancoAngeli.

A. Salvini (2007), Identità e tendenze del volontariato in Toscana, Firenze, Cesvot.

F. Susi (a c. di) (2007), Il volontariato nel Lazio. Identità, bisogni, caratteri, Roma, Carocci.

S. Licursi, G. Marcello (2008), «Pezzi di volontariato. Una ricerca empirica sulle associazioni di volontariato calabresi», Quaderni di Sociologia, LII, 47, pp. 35-67.

L. Antoni, S. Mele (2009), Le associazioni di volontariato in Toscana: caratteri strutturali e valenza occupazionale, Firenze, IRPET.

CESIAV, CSV Aurora (a c. di) (2009), Volontariato, Terzo Settore e questione meridionale oggi, Crotone, CSV Aurora.

R. Guidi (a c. di) (2009), Una promessa mantenuta? Volontariato, servizi pubblici, cittadinanza in Toscana, 2 Voll., n. 45, Firenze, Cesvot. 


\section{Questioni normative}

\subsection{La legge-quadro nazionale, evoluzione della disciplina e proposte di modifica}

AA.VV. (1991), «Disegno di legge sul volontariato proposto dalla Commissione Affari Costituzionali del Senato», Impresa Sociale, 1, pp. 42-47.

D. Venturini (1991), «La legge sul volontariato», Bambino Incompiuto, 3, pp. 141-149.

A. Bassi (1992), «Problemi e prospettive della nuova normativa relativa al volontariato e alle cooperative sociali», Autonomie Locali e Servizi Sociali, XV, 3, pp. 423-440.

G. Dente (1992), «Legge quadro sul volontariato e norme attuative: prime riflessioni», Impresa Sociale, 5, pp. 39-43.

G. Nervo (1992), «La Legge 266/91 e il ruolo politico del volontariato», Impresa Sociale, 5, pp. 36-39.

D. Rei (1992), «Dopo la legge: La "terza fase del volontariato"», Animazione sociale, XXII, 4, pp. 13-24.

L. Bruscuglia (a c. di) (1993), La Legge 266/91 sul volontariato: analisi e commento giuridico, Padova, CEDAM.

I. Colozzi (1993), «La legge quadro sul volontariato: problemi e prospettive», Impresa Sociale, 10, pp. 11-13.

G. Dal Ferro, E. Alecci, L. Pilon, S. Lepri (1993), Prospettive della legge sul volontariato, Vicenza, Rezzara.

G. Mariconda (1993), «Volontariato e cooperazione sociale. Le organizzazioni non profit nell'ordinamento giuridico», Quaderni di Volontariato, 2, Roma, FIVol.

G.P. Manganozzi (1993), Guida al volontariato italiano, Dit Dizionario tematico delle leggi (anni 1990-I ${ }^{\circ}$ Quadrimestre 1993), Torino, S.E.I.

L. Boccacin (1994), «L'impatto della legge 266/91 sull'azione volontaria. Elementi di riflessione da una indagine empirica», «Politiche Sociali e Servizi, 1, pp. 7-38.

D. Rei (1994), Note intorno alla legge quadro sul volontariato, in B. Cattarinussi (a c. di), Altruismo e solidarietà. Riflessioni su prosocialità e volontariati, Milano, FrancoAngeli, pp. 289-307.

G. Ponzanelli (1995), «Quali regole giuridiche per il terzo settore?», Non profit, IV, 1, pp. 549-554, poi in I. Colozzi (a c. di), Terzo settore e sviluppo civile: verso una 'regolazione promozionale', Milano, FrancoAngeli, 1997.

P. Benesperi et al. (1996), 266/91. Manuale sintetico per l'applicazione della legge 266/91 e della legislazione regionale della Toscana sul volontariato, Firenze, Regione Toscana.

L. Boccacin (1996), «Le organizzazioni di volontariato e la legge-quadro n. 286-91», Aggiornamenti sociali, XLVII, 1, pp. 39-51.

P. Donati, A.M. Maccarini, S. Stanziani (1996), L'associazionismo sociale oltre welfare State: quale regolazione?, Milano, FrancoAngeli.

P. Sciumè (1996), Come incrementare il terzo settore. Una proposta di legge innovativa: aspetti economici e giuridici, in G.M. Comolli, R. Garbagnati (a c. di), Volontariato verso il 2000. La solidarietà che diventa protagonista, Milano, FrancoAngeli.

P. Springhetti (a c. di) (1996), Volontariato e terzo settore. Quale legislazione? I problemi della regolamentazione e promozione del non profit in Italia, Roma, FIVol.

L. Boccacin (1997), La legge 266/91: il fronte delle organizzazioni e quello dei volontari. Elementi di riflessione da una ricerca, in I. Colozzi (a c. di), Terzo settore e sviluppo civile: verso una 'regolazione promozionale', cit., pp. 97-111.

E. Rossi (1997), «Privato sociale: evoluzione e limiti della legislazione italiana», Animazione Sociale, XXVII, 1, pp. 9-20. 
AA.VV. (1998), Lo stato di attuazione del D.M. 21/11/91 e successive modifiche, n. 1, Firenze, Cesvot.

C. Guccinelli, R. Podestà (1998), Terzo settore, Europa e nuova legislazione italiana sulle Onlus, n. 4, Firenze, Cesvot.

N. Lipari (1998), Per una disciplina del Terzo Settore. Proposta per una revisione della legislazione vigente, Roma, FIVol.

G. Nervo (2000), «La legge sul volontariato va cambiata?», Animazione Sociale, XXX, 6-7, pp. 86-89.

A. Fossati (2001), «Oltre lo Stato sociale: la legge-quadro sul volontariato», Aggiornamenti sociali, XLII, 12, pp. 821-828.

L. Saccone, E. Spagnoletti Zeuli (a c. di) (2001), Il volontariato tra legge 266/91 e authority. Atti del Convegno Nazionale di brescia del 24-25-26- Novembre 2000 e lavori preparatori, Milano, Fondazione Cariplo.

S. Nocera (2002), «Spazi e prospettive del volontariato culturale e professionale nelle Leggi 266/91 e 328/00», Studi Zancan: Politiche e Servizi alle Persone, 5, pp. 50-55.

G. Devastato (2004), La legge 328 e il volontariato, Roma, Centro Servizi per il Volontariato nel Lazio.

E. Rossi (2009), «Quale riforma legislativa per il volontariato?», Aretè, 3, pp. 105-115.

E. Rossi (a c. di) (2009), «Proposte per una riforma organica della legislazione sul Terzo settore», Aretè, suppl., pp. 5-53.

\subsection{Le leggi regionali ed ordinamenti locali}

Anci-Upi, Centro Nazionale Volontariato, Fondazione E. Zancan (1991), Partecipazione ed efficienza: il ruolo del volontariato negli statuti dei comuni e delle province, Roma, 6.2.1991, Lucca, Centro Nazionale Volontariato.

Corghi C. (a c. di) (1991), Volontariato e associazionismo di fronte alla legge di riforma delle autonomie locali, Lucca, Fondazione E. Zancan, Anci, Comune Padova, Centro Nazionale Volontariato.

AA.VV. (1992), Volontariato ed enti locali, Roma, Ed. Autonomie.

A. Bassi (1993), «Le leggi regionali sul volontariato: un'analisi sociologica», La Ricerca sociale, XLIX, poi in I. Colozzi (a c. di), Terzo settore e nuove politiche sociali in Italia e in Europa, Milano, FrancoAngeli, 1994.

L. Colombini (1993), «Il volontariato nelle leggi regionali nelle proposte e nei fatti», Animazione Sociale, XXIII, 3, pp. 69-70.

A. Bassi (1994), «Il volontariato nella legislazione regionale dopo la legge n. 266/1991», Autonomie locali e servizi sociali, XVII, 1, pp. 71-154.

Regione Lombardia (1994), «Regione Lombardia: la legge sul volontariato», Prospettive Sociali e Sanitarie, 2, pp. 18-21.

V. Cremoncini (a c. di) (1995), «Norme regionali applicative della legge n. 266/91», Servizi Sociali, 4, pp. 63-65.

P. Benesperi et al. (1996), 266/91. Manuale sintetico per l'applicazione della legge 266/91 e della legislazione regionale della Toscana sul volontariato, Firenze, Regione Toscana.

E. Gualandi et al. (1996), Volontariato e governo locale. I percorsi della solidarietà, Atti Del Convegno, Verona 21-23.10.1994, Roma, Edizioni delle Autonomie Locali.

AA.VV. (1998), Volontari e politiche sociali: La Legge regionale 72/97, n. 2, Firenze, Cesvot. 
M. Donatelli (1998), «Le leggi regionali in materia di volontariato e di cooperazione sociale», Politiche Sociali, 6, pp. 57-72.

F. Rao (1999), «L'attuazione della legge quadro sul volontariato nelle normative regionali», Servizi sociali, 5, pp. 37-68.

F. Dal Canto (2009), «Il volontariato nell`epoca delle autonomie», Aretè, 3, pp. 116-128.

G. Costa (a c. di) (2009), La solidarietà frammentata. Le leggi regionali sul welfare a confronto, Milano, Bruno Mondadori.

\subsection{Aspetti civilistici e fiscali}

G.M. Colombo (1993), Le organizzazioni di volontariato: aspetti civilistici e fiscali, Milano, Giuffrè.

V. Pinto (a c. di) (1996), Volontariato e fisco, Roma, FIVol.

C. Borzaga (1997), «Fisco per il non profit: di cosa tener conto nei decreti legislativi», Impresa Sociale, XXXII, pp. 2-6.

F. Angeloni (1999), Aspetti civilistici degli enti non profit e delle attività di volontariato nel diritto positivo vigente, Napoli, Edizioni scientifiche italiane.

L. Tavazza et al. (1998), Studi e proposte per il riordino della disciplina tributaria degli enti non profit, Roma, FIVol.

S. Ragghianti (a c. di) $\left(2000,2002^{2}, 2005^{3}\right)$, Raccolta normativa commentata. Leggi fiscali $e$ volontariato, n. 10, n. 16, n. 26, Firenze, Cesvot.

S. Ragghianti (2004), Le dichiarazioni fiscali degli Enti non Profit, n. 20, Firenze, Cesvot.

G.P. Manganozzi, A. Affanni (2005), Volontariato, terzo settore e politiche sociali in Italia: dizionario tematico delle leggi: DIT4 1975-2005, Roma, Editoriale italiana.

S. Lemmetti, R. Bemi (2009), Le donazioni al volontariato. Agevolazioni fiscali per i cittadini e le imprese, n. 44, Firenze, Cesvot.

S. Ricci, L. Corbella (2010), «Trattamento fiscale delle attività svolte da un'organizzazione di volontariato», Terzo Settore, 5, pp. 38-41.

E. Rossi (2010), «Approvate le Linee guida sui registri per il volontariato», Terzo Settore, 2, pp. 1-16.

\section{Il volontariato: questioni concettuali e riflessioni}

\subsection{Il volontariato e il terzo settore}

B. Gui (1991), «Le organizzazioni mutualistiche e senza fini di lucro. Un approccio unificato al Terzo settore», Stato e Mercato, 31, pp. 143-157.

B. Gui (1991), Il ruolo delle organizzazioni mutualistiche e senza fine di lucro, in C. Borzaga (a c. di), Il terzo sistema. Una nuova dimensione della complessità economica e sociale, Padova, Fondazione E. Zancan, pp. 57-66.

L. Boccacin (1993), La sinergia della differenza. Un'analisi sociologica del Terzo settore in Italia, Milano, FrancoAngeli.

A. Bassi (1994), «Il Terzo settore in Italia. Uno sguardo d'insieme», Autonomie locali e servizi sociali, XVII, 2, pp. 257-273.

I. Colozzi (1994), «Le specificità del volontariato nel contesto del terzo settore», Impresa Sociale, XVIII. 
Mo.v.i. (1994), Socializzare il territorio. Oltre l'emarginazione. I nuovi itinerari di autorganizzazione e di impegno del volontariato, Salerno, Movimento di Volontariato Italiano.

G. Bonini et al. (a c. di) (1995), Rapporti fra istituzioni non profit e associazioni di volontariato che le hanno promosse, Convegno di Fie' Allo Sciliar (Bz), 15-19.5.1995, Padova, Fondazione E. Zancan.

G.P. Barbetta (a c. di) (1996), Senza scopo di lucro. Dimensioni economiche, legislazione e politiche del settore non profit in Italia, Bologna, il Mulino.

G.P. Barbetta (1996), «Senza scopo di lucro. Un'analisi del Terzo settore in Italia», Prospettive Sociali e Sanitarie, 14, pp. 7-12.

L. Boccacin, G. Rossi (1996), Il volontariato, in P. Donati (a c. di), Sociologia del terzo settore, Roma, Nuova Italia Scientifica, pp. 173-194.

D. Bramanti (1996), «Il volontariato oggi in Italia. Quale posto nel Terzo Settore?», Studi di Sociologia, 3, pp. 241-257.

V. Cremoncini (1996), «Nota storica sula evoluzione della cultura e della evoluzione del volontariato», Servizi Sociali, 4, pp. 56-63.

C. Ranci (1996), «Non solo nonprofit», Impresa Sociale, XXV, pp. 21-28.

E. Ranci Ortigosa (1996), Attualità e futuro del Terzo Settore, in G.M. Comolli, R. Garbagnati (a c. di), Volontariato verso il 2000, cit.

S. Ristuccia (1996), Volontariato e fondazioni. Fisionomie del settore non profit, Rimini, Maggioli.

L. Boccaccin (1997), Terzo Settore. Mille volti del caso italiano, Milano, Vita e Pensiero.

C. Borzaga (1997), «Verso il riconoscimento del non profit: ma quale non profit?», Volontariato oggi, 3, 1, pp. 17-26.

P. Donati (1997), L'analisi sociologica del terzo settore: introdurre la distinzione relazionale terzo settore/privato sociale, in G. Rossi (a c. di), Terzo settore, stato e mercato nella trasformazione delle politiche sociali in Europa, Milano, FrancoAngeli, pp. 255-295.

G. Fiorentini (1997), Organizzazioni nonprofit e di volontariato, Milano, EtasLibri.

M. Ambrosini (1999), Il terzo settore come fenomeno sociale, in Id. (a c. di), Tra altruismo e professionalità. Terzo settore e cooperazione in Lombardia, cit., pp. 15-59.

F. Belletti (1999), «I volti della gratuità: alcune riflessioni in margine a un seminario di studio», Politiche Sociali e Servizi, 2, pp. 243-249.

M. Bottaccio (a c. di) (1999), Tutti al centro. Volontariato e Terzo settore in un paese normale, Roma, Minimum fax.

A. Cecconi (1999), Etica della gratuità: potenzialità diffusiva ed elemento qualificante il volontariato, Padova, Fondazione E. Zancan.

E. Ranci Ortigosa (1999), «Non profit e volontariato», Prospettive Sociali e Sanitarie, 11, pp. $1-2$.

N. Zamaro (1999), «Il volontariato organizzato. Quadro nazionale e tipologie organizzative emergenti», Prospettive Sociali e Sanitarie, 11, pp. 8-12.

L. Boccacin, D. Bramanti (a c. di) (2000), «Dare, ricevere, fidarsi. Cosa c'è di nuovo nel circuito del dono», Sociologia e Politiche Sociali, 2, pp. 119-154.

C. Borzaga (2000), «Gli attori del Terzo settore: ruoli e funzioni», Studi Zancan: Politiche e Servizi alle Persone, 2, pp. 29-45.

L. Bruscuglia, E. Rossi (a c. di) (2000), Terzo settore e nuove categorie giuridiche: le organizzazioni non lucrative di utilità sociale, Milano, Giuffré.

C. Cipolla (a c. di) (2000), Il co-settore in Italia. L'associazionismo pro-sociale tra logica di confine e logica corelazionale, Milano, FrancoAngeli.

M. La Rosa (a c. di) (2001), Le organizzazioni nel nuovo welfare. L'approccio sociologico pubblico, privato, sociale. Cooperazione e non profit, Rimini, Maggioli. 
G. Pasini (2001), «Il ruolo del terzo settore», Studi Zancan: Politiche e Servizi alle Persone, 2, pp. 81-87.

L. Boccacin, G. Rossi (2003), «Il terzo settore in Italia: le indicazioni emergenti dalla riflessione sociologica», Non profit, IX, 2, pp. 311-334.

C. Arcidiacono, I. Di Napoli, F. Procentese (2004), Tipologie associative e risorse, tra partecipazione ed organizzazione, in C. Arcidiacono (a c. di), Volontariato e legami collettivi. Bisogni di comunità e relazione reciproca, Milano, FrancoAngeli, pp. 76-106.

P. Donati (2004), Esplorare una galassia: il privato sociale come fenomeno emergente, in P. Donati, I. Colozzi (a c. di), Il privato sociale che emerge: realtà e dilemmi, Bologna, il Mulino, pp. 21-54.

G. Rossi, L. Boccacin (2004), Le culture e le pratiche del volontariato in Italia, in P. Donati, I. Colozzi (a c. di), Il terzo settore in Italia: culture e pratiche, Milano, FrancoAngeli, pp. 71-107.

G. Rossi, I. Colozzi (2004), «Cosa è il terzo settore: il contributo sociologico», Non profit, X, 1, pp. 9-15.

L. Boccacin (2005), Third Sector and Social Partnership in Italy. A Sociological Perspective, Milano, Vita e Pensiero.

L. Boccaccin, G. Rossi (2006), Le identità del volontariato italiano. Orientamenti valoriali e stili di intervento a confronto, Milano, Vita e Pensiero.

N. Nappo (2007), «Le determinanti del volontariato: una rassegna della letteratura teorica ed empirica», Non Profit, 3, pp. 659-685.

G. Nervo (2008), «L'etica del volontariato», Studi Zancan: Politiche e Servizi alle Persone, 9, 6, pp. 24-33.

R. Prandini (2008), L'autodifferenziazione del Terzo settore: ipotesi sull'emergere e l'istituzionalizzarsi di diverse culture organizzative e di nuove modalità di leadership, in I. Colozzi, R. Prandini (a c. di), I leader del terzo settore. Percorsi biografici, culture e stili di leadership, Milano, FrancoAngeli.

S. Longhi (2009), «La razionalità rispetto al valore nelle forme postmoderne di azione sociale: 1'esempio del terzo settore», Non profit, 2, pp. 81-105.

\subsection{Riflessioni sulle trasformazioni del volontariato}

F. Garelli (1992), «Il volontariato in Italia. Una forza o una debolezza?», il Mulino, XLI, 4, pp. 740-754.

AA.VV. (1993), Non eroi, ma cittadini. Volontariato, istituzioni, impresa, Roma, FIVol.

P. De Nardis (1993), «Regolazione istituzionale e sfida delle nuove etiche: il caso del volontariato in Italia», Sociologia e ricerca sociale, XIIL, pp. 5-45.

L. Boccacin (1994), «L'azione solidaristica organizzata: il "terzo settore” in Italia», Aggiornamenti sociali", XLV, 1, pp. 25-40.

C. Mongardini, M. Ruini (a c. di) (1994), Volontariato e nuove forme di solidarietà nella società contemporanea, Roma, La Goliardica.

L. Bianco, G. Tallone (1996), «Il gusto e la responsabilità dell'abitare. Presente e futuro del volontariato», Animazione Sociale, XXVI, 5, pp. 21-30.

L. Boccacin (1996), La funzione sociale del volontariato, in G.M. Comolli, R. Garbagnati (a c. di), Volontariato verso il 2000, cit., pp. 38-57.

M. Campedelli et al. (1996), «Dove va il volontariato?», Politiche Sociali, 1, pp. 5-21.

C. Degiacomi, M.R. Gilliavod (a c. di) (1996), Volontariato, Volontariati. Guida Ragionata, Torino, Edizioni Radionotizie.

P. Donati (1996), «I beni relazionali del Terzo Settore», Impresa Sociale, 29, pp. 19-23. 
P. Donati (1996), «Lo sviluppo delle organizzazioni di Terzo Settore nel processo di modernizzazione», Studi di Sociologia, 2, pp. 103-127.

P. Donati (1996), Volontariato verso il 2000: nuovi bisogni e prospettive, in Comolli G.M., R. Garbagnati (a c. di), Volontariato verso il 2000, cit., pp. 22-26.

B. Frediani (1996), «Il volontariato tra bisogno di sicurezza e solidarietà», Animazione Sociale, XXVI, 5, pp. 33-39.

A. Lulli (1996), «Il fenomeno delle nonprofit organization», Non profit, 3, p. 273.

P. Marchesi (1996), Il volontariato ispirato dalla solidarietà ma sostenuto dall'intelligenza, in G.M. Comolli, R. Garbagnati (a c. di), Volontariato verso il 2000, cit.

G. Vittadini (a c. di) (1997), Il Non Profit dimezzato, Milano, ETAS Libri.

A. Palmonari (1997), «Gratuità imperfetta», Rivista del Volontariato, 5, pp. 6-7.

P. Dal Dosso (1998), «Il Volontariato», Politiche Sociali, 1-2.

R. Frisanco (1998), «Il volontariato sociale in Italia: il presente tra passato e il futuro», Rivista del Volontariato, 6, p. 40.

L. Tavazza (1998), Il volontariato nella transizione. Le prospettive e le sfide fondamentali, Roma, FIVol.

M.E. Martini (1998), Evoluzione del volontariato: le ragioni, gli aspetti positivi e quelli problematici, Padova, Fondazione E. Zancan.

A. Matacena (a c. di) (1998), Aziende non profit. Scenari e strumenti per il Terzo settore, Milano, EGEA.

G. Nervo (1999), Il volontariato agente di cambiamento, Padova, Fondazione E. Zancan.

L. Tavazza (1999), La realtà del volontariato oggi, Padova, Fondazione E. Zancan.

E. Alecci et al. (2000), Dove va il volontariato?, Castelplanio (An), Gruppo Solidarietà.

G.P. Barbetta (2000), Il settore nonprofit italiano. Occupazione, welfare, finanziamento e regolazione, Bologna, il Mulino.

C. Borzaga, L. Fazzi (2000), Azione volontaria e processi di trasformazione del settore nonprofit, Milano, FrancoAngeli.

P. Dal Dosso (2000), «L'anno di volontariato sociale: elementi per una valutazione», in Studi Zancan: Politiche e Servizi alle Persone, 1, pp. 90-97.

P. Donati, I. Colozzi (a c. di) (2001), Generare «il civile»: nuovi scenari nella società italiana, Bologna, il Mulino.

C. Cattaneo (a c. di) (2001), Terzo settore, nuova statualità e solidarietà sociale, Milano, Giuffrè.

L. Fazzi (2001), «L'evoluzione del settore nonprofit in Italia: rottura o continuità nella riforma del welfare?», Sociologia e Politiche Sociali, XI, 3, pp. 43-67.

G. Tallone (2001), «Parole chiave per il volontariato», Animazione Sociale, XXXI, 4, pp. 1928.

A. Paltrinieri (2001), «La globalizzazione come sfida all'identità del volontariato», Tendenze nuove, 4 , pp. 303-312.

L. Fazzi (2002), «L'incerto destino del settore nonprofit in Italia», Politiche Sociali e Servizi, 1, pp. $35-55$.

G. Nervo (2002), «Ha un futuro il volontariato?», Studi Zancan: Politiche e Servizi alle Persone, 4, pp. 28-36.

D. Rei (2002), «Verso il sistema del volontariato: mondo chiuso o universo in espansione?», Animazione sociale, XXXII, 2, pp. 92-96.

G. Antonelli, M. Nosvelli (2003), «Opportunità e problemi aperti delle organizzazioni non profit», Non profit, IX, 2, pp. 181-226.

U. Ascoli (2003), «Le sfide del non profit italiano», Non profit, IX, 2, pp. 295-310. 
M. Serra (2003), Il protagonismo del Terzo Settore, in G Magistrali (a c. di), Il futuro delle politiche sociali in Italia. Prospettive e nodi critici della legge 328/2000, Milano, FrancoAngeli.

M. Ambrosini (2005), Scelte solidali. L'impegno per gli altri in tempi di soggettivismo, Bologna, il Mulino.

C. Buizza (2005), «Sfide e prospettive del volontariato», Prospettive Sociali e Sanitarie 12, pp. 4-8.

R. Frisanco (2006), Un fenomeno con tanti più e qualche campanello d'allarme, in Osservatorio Nazionale per il Volontariato, Ministero del Lavoro e delle Politiche Sociali, Rapporto Biennale sul Volontariato in Italia 2005, Roma, pp. 17-42.

R. Frisanco (2009), «Volontariati: trend e prospettive», Impresa Sociale, XIX, 4, pp. 21-42.

A. Salvini (2009), «Identità e trasformazioni del volontariato. Riflessioni alla luce del caso toscano», Areté, II, 2, pp. 97-111.

A. Salvini (2010), Il volontariato oltre il Welfare State, in M.A. Toscano (a c. di), ZoonPolitikon 2010. II - Politiche sociali e partecipazione, Firenze, Le Lettere, pp. 41-56.

\section{Il volontariato e i sistemi sociali}

\subsection{Il volontariato come 'terzo sistema'}

S. Lepri (1991), Il terzo sistema: una nuova dimensione della complessità economica e sociale, in C. Borzaga (a c. di), Il terzo sistema. Una nuova dimensione della complessità economica e sociale, cit., pp. 19-36.

D. Preite (1991), Le caratteristiche essenziali delle iniziative di terzo sistema, in C. Borzaga (a c. di), Il terzo sistema. Una nuova dimensione della complessità economica e sociale, cit., pp. 37-56.

C. Ranci, U. De Ambrogio, S. Pasquinelli (1991), Identità e servizio. Il volontariato nella crisi del Welfare, Bologna, il Mulino.

U. Ascoli (1992), «L'azione volontaria nei sistemi di welfare», Polis, 3, pp. 429-436.

U. Ascoli, S. Pasquinelli (a c. di) (1993), Il welfare mix. Stato sociale e terzo settore, Milano, FrancoAngeli.

G. Rossi (1993), «La relazione tra pubblico e volontariato: quali implicazioni per la politica sociale», La Ricerca Sociale, XLIX, pp. 163-177, poi in I. Colozzi (a c. di), Terzo settore e nuove politiche sociali in Italia e in Europa, cit.

L. Boccacin (1994), Solidarietà e terzo settore, in B. Cattarinussi (a c. di), Altruismo e solidarietà. Riflessioni su prosocialità e volontariati, cit.

P. Natale (1994), Forme e finalità dell'azione solidaristica, in B. Cattarinussi (a c. di), Altruismo e solidarietà. Riflessioni su prosocialità e volontariati, cit.,

C. Ranci (1994), «Il terzo settore nelle politiche di Welfare in Italia: le contraddizioni di un mercato protetto», Stato e Mercato, 42, pp. 323-364.

C. Ranci (1994), Altruismo e reciprocità: due modelli di solidarietà a confronto, in B. Cattarinussi (a c. di), Altruismo e solidarietà. Riflessioni su prosocialità e volontariati, cit., pp. 49-55.

C. Ranci, A. Vanoli (1994), Beni pubblici e virtù private. Il Terzo settore nelle politiche di Welfare, Perugia, Fondazione Olivetti.

M. Ambrosini (1995), «Crisi dello stato sociale. Terzo settore e volontariato», in Aggiornamenti Sociali, LVI, 11, pp. 707-719. 
V. Cotesta, E. Bartocci (a c. di) (1995), Il welfare italiano: teorie, modelli e pratiche dei sistemi di solidarietà sociale, Roma, Donzelli.

U. Ascoli (1996), «Welfare Mix e coprogettazione dei servizi», Quaderni, II, pp. 19-24,

U. Ascoli (1996), Soggetti pubblici e organizzazioni del "terzo settore": appunti sul welfare mix in Italia, in A. Fadda (a c. di), Interrelazione volontaria e solidarietà nella società complessa, Milano, FrancoAngeli, pp. 161-168.

C. Borzaga, G. Fiorentini, A. Matacena (a c. di) (1996), Non profit e sistemi di welfare. Il contributo dell'analisi economica, Roma, NIS.

P. Donati (1996), «Lo sviluppo delle organizzazioni di Terzo Settore nel processo di modernizzazione», Studi di Sociologia, 2, pp. 103-127.

M. Gorgoni (1996), «Le organizzazioni del Terzo settore nel rinnovamento dello stato sociale», Volontariato Oggi, 4.

S. Beretta (1997), «Qualità dello stato sociale e imprese "non profit": spunti di riflessione», Vita e Pensiero, LXXX, 5, pp. 342-352.

F. Meloni (1996), Idioti o solidali? Stato sociale e politiche sociali tra liberismo e assistenzialismo, non profit e volontariato, Terlizzi (Ba), Ed. Insieme.

S. Stanzani (1996), «La specificità relazionale del terzo settore», Impresa sociale, XXV, pp.15-20.

I. Colozzi (1997), Il principio di sussidiarietà come principio regolatore delle politiche sociali, in G. Rossi (a c. di), Terzo settore, stato e mercato nella trasformazione delle politiche sociali in Europa, cit., pp. 24-33.

F. Scalvini (1997), «Politiche sociali attive per il welfare di comunità», Impresa Sociale, XXXV, 5, pp. 6-14.

L. Tavazza (1997), «Volontariato e stato sociale in crisi», Rivista del Volontariato, 4, pp. 3639.

I. Colozzi (1998), «La sussidiarietà come principio regolatore del nuovo stato sociale», Sociologia e Politiche Sociali, X, 1, pp. 53-77.

O. De Leonardis (1998), In un diverso welfare: sogni e incubi, Milano, Feltrinelli.

D. Rei (1998), «Il terzo settore nella transizione del welfare», Animazione Sociale, XXVIII, 1 , pp. $20-26$.

S. Stanzani (1998), La specificità relazionale del Terzo settore, Milano, FrancoAngeli.

G. Vittadini (a c. di) (1998), Sussidiarietà: la riforma possibile, Milano, ETAS Libri.

U. Ascoli (a c. di) (1999), Il Welfare futuro. Manuale critico del Terzo Settore, Carocci, Roma.

L. Fazzi, E. Messora (a c. di) (1999), Modelli di welfare mix, Milano, FrancoAngeli.

G. Nervo (1999), «Volontariato: servizio o dono?», Politiche Sociali, 2, pp. 15-20.

C. Ranci (1999), Oltre il Welfare State. Terzo settore, nuove solidarietà e trasformazioni del welfare, Bologna, il Mulino.

A. Bassi (2000), Dono e fiducia. Le forme della solidarietà nelle società complesse, Roma, Edizioni Lavoro.

I. Colozzi (2000), «La cultura della solidarietà nella società italiana contemporanea», Sociologia e Politiche Sociali, X, 1, pp. 158-169.

A. Prezioso (2000), «Il Terzo settore nella realizzazione dello stato sociale», Studi Zancan: Politiche e Servizi alle Persone, 2, pp. 7-28.

A. Santuari, G. Cittadini (2000), «La sussidiarietà: una nuova forma di partnership tra pubblico e privato», Non profit, 2, pp. 121-125.

L. Fazzi (2001), «L'evoluzione del settore nonprofit in Italia: rottura o continuità nella riforma del welfare?», in Sociologia e Politiche Sociali, XI, 3, pp. 43-67. 
F. Cafaggi (a c. di) (2002), Modelli di governo, riforma dello stato sociale e ruolo del Terzo settore, Bologna, il Mulino.

P. Costa (2002), Oltre lo Stato: teorie pluralistiche del primo Novecento, in M. Bortolini (a c. di), «Agire associativo e sfera pubblica», Sociologia e Politiche Sociali, XIII, 5, pp. 11-36.

A. Quadrio Curzio, G. Bertoni (a c. di) (2002), Non profit e sussidiarietà, Milano, FrancoAngeli.

L. Boccacin (2003), Il Terzo settore tra le generazioni. Un'analisi delle relazioni tra i soggetti del welfare plurale, Milano, Vita e Pensiero.

E. Pavolini (2003), Le nuove politiche sociali. I sistemi di welfare fra istituzioni e società civile, Bologna, il Mulino.

G. Vittadini (a c. di) (2003), Liberi di scegliere: dal welfare state alla welfare society, Milano, ETAS Libri.

P. Donati (2004), Alla ricerca del nucleo generativo del terzo settore, in P. Donati, I. Colozzi (a c. di), Il privato sociale che emerge: realtà e dilemmi, cit., pp. 9-17.

R. Frisanco (2005), «Volontariato e sussidiarietà», in Oggi Domani Anziani, 2, pp. 61-71.

F. Pizzolato (2005), «Partecipazione, sussidiarietà e ruolo del terzo settore», in Aggiornamenti Sociali, LXVI 1, pp. 11-22.

P. Venturi, N. Montanari (a c. di) (2005), Modelli e forme organizzative del decentramento: ruolo e sviluppo del terzo settore, Forlì, AICCON.

A. Turchini (2006), «Il ruolo e la funzione della sussidiarietà nei nuovi modelli di governance sociale: alcuni casi di studio», in «Osservatorio Isfol», XXVII, 1-2.

T. Vitale (2006), «A che cosa serve la sussidiarietà. Un criterio guida contro il carsismo istituzionale», Animazione Sociale, XXXVI, 5, pp. 20-28.

\subsection{Il volontariato e la cittadinanza}

Censis (1991), Associazionismo. I nuovi canali di consenso e di partecipazione, Milano, FrancoAngeli.

B. Tomai et al. (a c. di) (1991), Associazionismo, volontariato e nuova cittadinanza sociale, Cernusco sul Naviglio (Mi), Cens-Coop. Editrice Nuova Stampa.

P. Donati (1993), Democrazia e cittadinanza: al di là di un codice evolutivo problematico, in P. Donati, G.B. Sgritta (a c. di), Cittadinanza e nuove politiche sociali, Milano, FrancoAngeli, pp. 11-58.

T. Vecchiato, G. Pasini et al. (1995), «Volontariato e tutela dei diritti sociali», Servizi Socia$l i$, XXII, 1, Padova, Fondazione E. Zancan.

P. Donati (1997), La società civile in Italia, Milano, Mondadori.

M. Lucà (1997), «Futuro del no-profit e qualità del paese», Animazione Sociale, 27, 3, pp. 95-96.

G. Cotturri (1998), La cittadinanza attiva, Roma, FIVol.

A. Mutti (1998), Capitale sociale e sviluppo. La fiducia come risorsa, Bologna, il Mulino.

G. Pasini et al. (1999), Seminario di Ricerca "Il volontariato strumento di garanzia e rinnovamento di diritti nell'attuale società in trasformazione", Padova, Fondazione E. Zancan.

M. Diani (2000), «Capitale sociale, fiducia istituzionale e azione volontaria», Rivista Italiana di Scienze Politiche, XXX, 3, pp. 475-511.

IREF-ACLI (2000), L'impronta civica: le forme di partecipazione sociale degli Italiani, Roma, Edizioni del Lavoro.

G.P. Barbetta (2002), Nonprofit. Il nuovo volto della società civile, Bologna, il Mulino. 
P. Donati, I. Colozzi (a c. di) (2002), La cultura civile in Italia: fra stato, mercato e privato sociale, Bologna, il Mulino.

M. Lori (2002), Il "capitale sociale" delle province lombarde, in C. Caltabiano (a c. di), Lombardia solidale. Terzo settore e civismo in una regione in transizione, cit., pp. 67-84.

A. Ardigò (2003), «Riflessioni critiche e idee per gli sviluppi possibili del volontariato di advocacy», Politiche e Servizi alle Persone, 1, pp. 8-20.

C. Caltabiano (a c. di) (2003), Il sottile filo della responsabilità civica. Gli italiani e la sfera pubblica: VIII Rapporto sull'associazionismo sociale, Milano, FrancoAngeli.

P. Donati (2003), Associarsi e promuovere una società civile, in S. Stanzani (a c. di), Percorsi di civilizzazione. La cultura delle relazioni sociali nelle organizzazioni del terzo settore, Rimini, Maggioli, pp. 15-43.

M. Giordano (2003), «La funzione del volontariato di advocacy», Politiche e Servizi alle Persone, 1, pp. 20-28.

D. La Valle (2003), «Capitale sociale in Italia: 1'andamento della partecipazione associativa», Inchiesta, 139, pp. 130-143.

C. Arcidiacono (2004), I volontariati e la società civile in una prospettiva globale, in Id. (a c. di), Volontariato e legami collettivi. Bisogni di comunità e relazione reciproca, cit., pp. 248-266.

V. Cesareo (a c. di) (2004), I protagonisti della società civile, Soveria Mannelli, Rubettino.

C. De Luca (2004), Il volontariato per la formazione dell'uomo solidale, Soveria Mannelli, Rubettino.

N. De Piccoli (2004), Volontariato e partecipazione, in C. Arcidiacono (a c. di), Volontariato e legami collettivi. Bisogni di comunità e relazione reciproca, cit., pp. 173-187.

P. Donati (2004), Il ruolo societario delle associazioni sociali, in P. Donati, I. Colozzi (a c. di), Il privato sociale che emerge: realtà e dilemmi, cit., pp. 44-58.

M. Granelli (2004), «Advocacy del volontariato o volontariato d'advocacy?», Politiche e Servizi alle Persone, 3, pp. 58-69.

G. Lavanco, M. Mandalà (2004), Comunità e capitale sociale. Elementi per una riflessione, in C. Arcidiacono (a c. di), Volontariato e legami collettivi. Bisogni di comunità e relazione reciproca, cit., pp. 213-228.

E. Mebane Minou (2004), Capitale sociale e partecipazione politica, in C. Arcidiacono (a c. di), Volontariato e legami collettivi. Bisogni di comunità e relazione reciproca, cit., pp. 229-247.

G. Nervo (2004), «Il volontariato di promozione e tutela dei diritti», Studi Zancan: Politiche e Servizi alle Persone, 1, pp. 30-42.

A. Salvati (2004), Alla ricerca dell'altruismo perduto. Altruismo, cooperazione, capitale sociale, Milano, FrancoAngeli.

G. Moro (2005), Azione civica, Roma, Carocci.

L. Boccacin (2006), Il capitale sociale è polimorfo, in G. Rossi, Id. (a c. di), Il capitale sociale in un'organizzazione multilivello di terzo settore, Milano, FrancoAngeli, pp. 213-245.

P. Di Nicola (a c. di) (2006), Dalla società civile al capitale sociale. Reti associative e strategie di prossimità, Milano, FrancoAngeli.

P. Di Nicola (2006), Le dinamiche di costruzione del capitale sociale, l'esperienza dell'associazionismo nella realtà veronese, in $\mathrm{P}$. Donati, I. Colozzi (a c. di), Terzo settore e valorizzazione del capitale sociale in Italia: luoghi e attori, cit., pp. 167-190.

P. Donati (2006), Introduzione. La valorizzazione del capitale sociale in Italia: luoghi e atto$r i$, in P. Donati, I. Colozzi (a c. di), Terzo settore e valorizzazione del capitale sociale in Italia: luoghi e attori, cit., pp. 9-22. 
G. Rossi (2006), Capitale sociale e organizzazioni di terzo settore, in Id., Boccaccin L. (a c. di), Il capitale sociale in un'organizzazione multilivello di terzo settore, Milano, FrancoAngeli, pp. 11-26.

R. Cartocci (2007), Mappe del tesoro. Atlante del capitale sociale in Italia, Bologna, il Mulino.

F. Sabatini (2007), «Un atlante del capitale sociale italiano», QA Rivista dell'Associazione Rossi Doria, 1, pp. 41-73.

I. Bartholini (2008), Il capitale sociale e la rete partecipativa, in I. Barholini (a c. di), Capitale sociale, reti comunicative e culture di partecipazione, Milano, FrancoAngeli, 2008, pp. 125-140.

I. Colozzi (2008), Il capitale sociale in prospettiva relazionale, in I. Barholini (a c. di), Capitale sociale, reti comunicative e culture di partecipazione, cit., pp. 25-38.

P. Di Nicola (2008), Capitale sociale e società civile: reti e strategie di prossimità, in I. Barholini (a c. di), Capitale sociale, reti comunicative e culture di partecipazione, cit., pp. 39-46.

S. Giusti, A. Caldelli (a c. di) (2008), La città competente. Il volontariato come percorso di formazione, n. 16, Firenze, Cesvot.

A. La Spina (2008), Capitale sociale e legalità debole, in I. Barholini (a c. di), Capitale sociale, reti comunicative e culture di partecipazione, cit., pp. 48-58.

A. Mosca (a c. di) (2008), Il volontariato e il nuovo welfare. Partecipazione, Legge 328/00, programmazione dei servizi in Lombardia, Milano, FrancoAngeli.

G. Panizza (2008), Il volontariato per la legalità, in «Volontariato Oggi», 24, 3, pp. 16-17.

L. Boccaccin (2009), «Rispondere ai bisogni creando legami sociali: il contributo del volontariato», Politiche Sociali e Servizi, XI, 1, pp. 9-40.

R. Guidi (a c. di) (2009), Una promessa mantenuta? Volontariato, servizi pubblici, cittadinanza in Toscana, 2 Voll., n. 45, Firenze, Cesvot.

E.M. Tacchi (2009), Il volontariato: tra scelte politiche, impegno sociale e funzioni di advocacy, Brescia, La scuola.

D. Cordaz (2010), Volontariato e coesione sociale, in Toscano M.A. (a c. di), ZoonPolitikon 2010. Politichesociali e partecipazione, Firenze, Le Lettere, pp. 93-110.

M. Villa (2010), Il Terzo settore ostacola o favorisce la partecipazione?, in M.A. Toscano (a c. di), Zoon politikon 2010, cit., pp. 75-92.

\subsection{Il volontariato, $i$ sistemi familiari ed educativolformativi}

R. Franzoni (a c. di) (1993), Volontariato e scuola. Solidarietà: il nuovo fronte dell'educazione civile, Roma, FIVol.

G. Rossi (2003), Le famiglie dei giovani-adulti volontari e la trasmissione tra le generazioni: stili relazionali a confronto, in L. Boccacin, E. Marta (a c. di), Giovani-adulti, famiglia e volontariato. Itinerari di costruzione dell'identità personale e sociale, Milano, Unicopli, pp. $65-96$.

E. Marta (2004), «Matrice familiare e storia intergenerazionale nella scelta di impegno nel volontariato dei giovani», Sociologia e politiche sociali, 7, 2, pp. 77-91.

E. Marta, M. Pozzi (2004), Generatività e volontariato: quale connessione?, in C. Arcidiacono. (a c. di), Volontariato e legami collettivi. Bisogni di comunità e relazione reciproca, cit., pp. 188-212.

G. Rossi (2004), La trasmissione della prosocialità tra le generazioni: il caso delle famiglie dei giovani-adulti volontari, in L. Boccacin, G. Rossi (a c. di), «Stili partecipativi emergenti nel volontariato giovanile», Sociologia e politiche sociali, VII, 2, pp. 59-76. 
P. Donati, I. Colozzi, R. Prandini, L. Tonca (2006), Tre forme di capitale sociale tra famiglia e scuola: chi e come genera beni relazionali neri processi di socializzazione delle nuove generazioni?, P. Donati, I. Colozzi (a c. di), Terzo settore e valorizzazione del capitale sociale in Italia: luoghi e attori, cit., pp. 23-62.

I. Colozzi (2006), Conclusioni. Valorizzare il Terzo settore e la famiglia per valorizzare il capitale sociale, in P. Donati, I. Colozzi (a c. di), Terzo settore e valorizzazione del capitale sociale in Italia: luoghi e attori, cit., pp. 279-286.

A.M. Maccarini (2006), Crescita personale, cultura civile e capitale sociale nel Terzo settore: due casi nel sistema educativo, in P. Donati, I. Colozzi (a c. di), Terzo settore e valorizzazione del capitale sociale in Italia: luoghi e attori, cit., pp. 63-94.

S. Guglielmi, C. Buzzi (a c. di) (2007), Il volontariato a scuola. Esperienze di solidarietà tra educazione e formazione, Milano, FrancoAngeli.

G. Rossi, L. Boccacin, E. Carrà (2007), Il quadro di riferimento teorico e metodologico della ricerca, in G. Rossi, L. Boccacin (a c. di), Capitale sociale e partnership tra pubblico, privato e terzo settore. Vol I. Casi di buone pratiche nei servizi alla famiglia, Milano, FrancoAngeli.

P. Fradeani (2008), «A scuola di volontariato associativo», Rassegna dell 'istruzione, 4/5, pp. $15-25$.

F. Siringo (2010), «Volontariato e scuola nella sfida educativa», in «Psicologia di comunità», 1, pp. 111-127.

\subsection{Il volontariato e il sistema politico-amministrativo}

G.F. Barbetta (1991), I rapporti tra stato e terzo sistema, in C. Borzaga (a c. di), Il terzo sistema. Una nuova dimensione della complessità economica e sociale, cit., pp. 115-122.

N. Lipari (1991), «Quali rapporti tra volontariato e partiti politici?», Animazione Sociale, XXI, 5, pp. 10-16.

A. Papisca (1991), «Il volontariato, soggetto di democrazia qualitativa», Pace, diritti dell'uomo, diritti dei popoli, V, 1, pp. 43-48.

C. Saraceno (1991), Nuovi rapporti tra agire solidaristico e soggetto pubblico, in C. Borzaga (a c. di), Il terzo sistema. Una nuova dimensione della complessità economica e sociale, cit., pp. 105-110.

AA.VV., (1992) Volontariato ed enti locali, Roma, Ed. Autonomie.

G. Sanavio (1992), Organizzazioni di terzo sistema e correlazioni con le forme di gestione dei servizi. In particolare lo strumento delle convenzioni, Padova, Fondazione E. Zancan.

G. Sarpellon et al. (1992), «Verso un ruolo politico del volontariato», Servizi Sociali, XIX, 6, Padova, Fondazione E. Zancan.

E. Gualandi et al. (1996), Volontariato e governo locale. I percorsi della solidarietà, Atti Del Convegno, Verona, 21-23.10.1994, Roma, Edizioni delle Autonomie Locali.

S. de Götzen (1997), «Organizzazioni di volontariato: relazioni con le amministrazioni pubbliche», Le Regioni, XXV, 4, pp. 575-586.

A. Battistella (1998), «Convenzioni tra enti pubblici e organizzazioni non profit», Prospettive Sociali e Sanitarie, 7, pp. 6-8.

L. Leone (a c. di) (1998), 10 buoni casi di partnership tra terzo settore ed enti locali, Roma, FORUM P.A.

M.E. Martini (1998), Rapporti tra soggetti pubblici e non profit: lo specifico del volontariato, in AA.VV., Volontari e politiche sociali: La Legge regionale 72/97, n. 2, Firenze, Cesvot, pp. 72-97. 
M. Musella (a c. di) (1998), Volontariato, politiche sociali e bilanci comunali, Napoli, Edizioni Athena.

L. Anfossi (1999), Le prospettive dell'accreditamento, Padova, Fondazione E. Zancan.

F. Dalla Mura (1999), Gli strumenti per l'integrazione istituzionale e operativa: protocolli, convenzioni, accordi e contratti di programma, Padova, Fondazione E. Zancan.

A. Migliorini (1999), Il rapporto tra volontariato e istituzioni, Padova, Fondazione E. Zancan.

A. Battistella (2000), «I problemi aperti nell'interazione pubblico/non profit», Prospettive Sociali e Sanitarie, 15-16, pp. 1-3.

A. Castegnaro (2002), «Il volontariato ai tavoli di concertazione: istruzioni per l'uso», Studi Zancan: Politiche e Servizi alle Persone, 1, pp. 40-61.

W. Nanni (2002), Bisogni che non trovano risposta e programmazione sociale: quale apporto dal volontariato?, Padova, Fondazione E. Zancan.

E. Vagnoni, E. Bracci (2002), «Quale ruolo per il volontariato nelle attività degli enti locali? Considerazioni da un' analisi empirica», La Finanza locale, XXII, 1, pp. 91-121.

F. Dalla Mura (2003), Pubblica amministrazione e non profit: guida ai rapporti innovativi nel quadro della Legge 328/2000, Roma, Carocci.

C. Buizza (2004), «Volontariato ed ente pubblico: quale policy», Prospettive Sociali e Sanitarie 20, pp. 8-10.

D. Bezzi (2005), Appalti, concessioni e convenzioni tra Enti Pubblici e terzo settore, Milano, Il Sole 24 ore.

P. Minetti (2005), Il rapporto tra gli enti locali e gli organismi no profit, Rimini, Maggioli.

C. Buizza (2006), «Aspettative di rete per un nuovo welfare: l'orizzonte dei rapporti volontariato-ente locale», Aggiornamenti Sociali, LVII, 4, pp. 311-320.

V. Andreani, A. Minà (a c. di) (2007), Amministrazioni pubbliche e volontariato: stato e prospettive, Soveria Mannelli, Rubbettino.

M. Villa (2008), La sfida della gratuità. Il volontariato a Brescia tra altruismo e istituzioni, Milano, FrancoAngeli.

G. Cangelosi (2010), «L'emergenza tra volontariato e pubblici poteri», Nuova rassegna di legislazione, dottrina e giurisprudenza, 84, 9/10, pp. 981-995.

\subsection{Il volontariato e il sistema economico}

C. Borzaga (1991), Terzo sistema e occupazione: la dimensione quantitativa, in C. Borzaga (a c. di), Il terzo sistema. Una nuova dimensione della complessità economica e sociale, Padova, cit., pp. 83-98.

S. Lepri (1991), «La natura economica dell'attività di volontariato: un'ipotesi di valutazione e di misurazione», Impresa Sociale, 1, pp. 35-41.

L. Boccacin (1993), «Volontariato ed organizzazioni non profit: la dimensione socioeconomica del terzo settore», La ricerca sociale, XLIX, pp. 215-241, poi in I. Colozzi (a c. di), Terzo settore e nuove politiche sociali in Italia e in Europa, cit.

R. Franzoni (a c. di) (1993), Volontariato ed economia. Tra profitto e solidarietà: lo sviluppo possibile, Roma, FIVol.

F. Mari (a c. di) (1994), Volontariato e impresa, Roma, FIVol.

G. Milanesi (1994), Volontariato, solidarietà, mondo del lavoro, Rassegna CNOS.

Centro Studi Cgm (1995), Il volontariato nelle cooperative sociali, Milano, Cgm.

S. Lepri (1995), «Il volontariato in cooperativa sociale: elemento di successo», Impresa Sociale, XXIV, pp. 13-27. 
S. Zamagni (1995), «Il settore non profit in un'economia post-industriale», Etica degli Affari e delle Professioni, 3, pp.17-22.

C. Borzaga (1996), «Terzo settore e occupazione: un binomio sostenibile», Impresa Sociale, XXVII, pp. 2-6.

A.M.A. Merlo (1996), Non profit nell'accezione economico-aziendale, in G.M. Comolli, R. Garbagnati (a c. di), Volontariato verso il 2000, cit.

S. Zamagni (1996), Il terzo settore in un'economia post-industriale, in G.M. Comolli, R. Garbagnati (a c. di), Volontariato verso il 2000, cit., pp. 22-26.

S. Zamagni (1996), «Se si afferma l’economia civile», Impresa Sociale, 29, pp. 29-32.

L. Boccacin (1997), Il mercato è un polo attrattore discriminante per il terzo settore? Alcune brevi note in merito a questo interrogativo, in G. Rossi (a c. di), Terzo settore, stato e mercato nella trasformazione delle politiche sociali in Europa, cit., pp. 110-114.

C. Borzaga, A. Bacchiega (1997), L'analisi economica delle organizzazioni non profit: limiti degli approcci tradizionali e possibili sviluppi, in G. Rossi (a c. di), Terzo settore, stato e mercato nella trasformazione delle politiche sociali in Europa, cit., pp. 237-254.

A. Orioli (a c. di) (1997), Lavorare nel no profit, Milano, Il Sole-24 Ore.

C. Travaglini (1997), Classificazione ed agevolazione delle aziende di terzo settore: un approccio economico-aziendale, in I. Colozzi (a c. di), Terzo settore e sviluppo civile: verso una 'regolazione promozionale', cit.

S. Zamagni (1997), Il settore non profit in un'economia post-industriale, in I. Colozzi (a c. di), Terzo settore e sviluppo civile: verso una 'regolazione promozionale', cit.

IREF-ACLI (1998), L'imprenditorialità solidale. Prospettive occupazionali e potenzialità di sviluppo dell'economia civile in Italia, Roma, Editoriale AESSE.

L. Tavazza (1998), «Volontariato e occupazione», Rivista del Volontariato, 9, p. 37.

S. Zamagni (1998), Non profit come economia civile, Bologna, il Mulino.

A. Gamba (1999), Le dimensioni economiche, in M. Ambrosini (a c. di), Tra altruismo e professionalità. Terzo settore e cooperazione in Lombardia, cit.

P. Tubaro (1999), Critica della ragion nonprofit: l'economia solidale è una truffa?, Roma, Derive Approdi.

M. Musella, S. D'Acunto (2000), Economia politica del nonprofit, Torino, Giappichelli.

M.L. Piga (2000), Imprenditori per profitto, imprenditori per solidarietà, Milano, FrancoAngeli.

S. Zamagni (2001), «Tra volontariato ed economia civile», Rivista della cooperazione, 4, pp. 39-53.

F. Archibugi (2002), L'economia associativa: sguardi oltre il Welfare state e nel postcapitalismo, Torino, Edizioni di Comunità.

C. Borzaga (2002), «Terzo settore e occupazione: tra missione sociale e politiche di sostegno», Quaderni di Eguaglianza \& Libertà, pp. 139-156.

R. Frisanco (2002), «L'occupazione nel volontariato», Terzo Settore, 11.

G. Marangoni (2002), Dal non profit all'economia civile, Vicenza, Ergon Edizioni.

F. Tei (2002), Scenari occupazionali del terzo settore in Lombardia, in C. Caltabiano (a c. di), Lombardia solidale. Terzo settore e civismo in una regione in transizione, cit.

S. Zamagni (2002), «Volontariato ed economia sociale: Quale rapporto», Studi Zancan: Politiche e Servizi alle Persone, 5, pp. 100-128.

S. Zamagni (2002), «Economia o Economie sociali?», Terzo Settore, 7.

S. Zamagni (2002), «L'economia civile», Terzo Settore, 9. 
C. Borzaga (2003), Il lavoro nel terzo settore, in G. Ferraro (a c. di), Sviluppo e occupazione nell'Europa federale. Itinerari giuridici e socioeconomici su Regioni e autonomie locali, Milano, Giuffrè, pp. 547-555.

C. Borzaga (2003), Il lavoro nelle organizzazioni nonprofit: una rassegna della letteratura empirica, in Id., M. Musella (a c. di), Produttività ed efficienza nelle organizzazioni nonprofit. Il ruolo dei lavoratori e delle relazioni di lavoro, Trento, Edizioni31, pp. 165-183.

G. Cerulli (2003), «Terzo settore e sviluppo economico: gli effetti su equità, efficienza e occupazione per il caso italiano», Impresa Sociale, 71-72, pp. 27-43.

E. Rossi (2004), «Sfide per il volontariato attore dello sviluppo locale», Studi Zancan: Politiche e Servizi alle Persone, 6, pp. 51-74.

M. Crescenzi, E. Bonacini, ASVI (a c. di) (2005), Guida internazionale alle professioni e al lavoro nel non profit, Bologna, EMI.

G. D'angelo (2005), Il perfetto non profit, Milano, Lupetti.

L. Bruni (2007), Reciprocità. Dinamiche di cooperazione, economia e società civile, in P. Donati (a c. di), Il capitale sociale. L'approccio relazionale, Milano, FrancoAngeli.

D. Fiorillo (2008), «Lavoro volontario: un'analisi 'cross'-sezionale sul dataset Multiscopo», Impresa Sociale, LXXVII, 2, pp. 119-140.

\section{Ambiti di intervento: le politiche sociali e socio-sanitarie}

P. Donati (1991), Il ruolo delle iniziative di «terzo sistema» nelle politiche sociali, in C. Borzaga (a c. di), Il terzo sistema. Una nuova dimensione della complessità economica e sociale, cit., pp. 67-82.

G. Rossi (1993), Welfare systems, servizi socio-sanitari e diritti di cittadinanza, in P. Donati, G.B. Sgritta (a c. di), Cittadinanza e nuove politiche sociali, cit., pp. 97-128.

S. Lepri (1993), «Le organizzazioni di volontariato possono gestire e vendere servizi sociali?», Impresa Sociale, 10, pp. 34-42.

P. David (1994), Pubblico e privato nel settore socio-assistenziale italiano, in R. De Vita, P. Donati, G.B. Sgritta (a c. di), La politica sociale oltre la crisi del welfare state, Milano, FrancoAngeli.

L. Dolazza (a c. di) (1994), Solidarietà al di là del muro, Milano, Vita e Pensiero.

L Fazzi (1994), «Affidamento di servizi sociali a agenzie non profit: problemi e prospettive», Economia Pubblica, 6, pp. 53-68.

F. Folgheraiter (1994), Integrazione di politiche sociali e lavoro sociale nelle comunità locali: la community care, in R. De Vita, P. Donati, G.B. Sgritta (a c. di), La politica sociale oltre la crisi del welfare state, cit.

G. Rossi (1994), Politiche sociali e volontariato, in R. De Vita, P. Donati, G.B. Sgritta (a c. di), La politica sociale oltre la crisi del welfare state, cit.

G.B. Sgritta (1994), Le trasformazioni della politica sociale dopo la crisi del welfare state, R. De Vita, P. Donati, G.B. Sgritta (a c. di), La politica sociale oltre la crisi del welfare state, cit.

L. Boccacin (1995), «Il volontariato organizzato: una risorsa strategica per i bisogni sociosanitari», Politiche Sociali e Servizi, 1, pp. 7-39.

C. Borzaga, B. Gui, M. Schenkel (1995), «Disoccupazione e bisogni insoddisfatti: il ruolo delle organizzazioni non profit», Quaderni di Economia del Lavoro, LII, pp. 205-233.

C. Marzotto (1995), «Essere volontari e lavorare con le famiglie con un membro in difficoltà», Politiche Sociali e Servizi, 2, pp. 235-253. 
S. Piazza (1995), «Le politiche per la salute: normative nazionali e regionali, l'intervento del volontariato», Servizi Sociali, 3, pp. 41-48.

L. Boccacin (1996), Terzo settore e politiche sociali, in A. Fadda (a c. di), Interrelazione volontaria e solidarietà nella società complessa, Milano, FrancoAngeli, pp. 173-180.

C. Bonfioli (1996), Qualità nel "volontariato sanitario", in G.M. Comolli, R. Garbagnati (a c. di), Volontariato verso il 2000 , cit.

C. Coppola (a c. di) (1996), Volontariato e giustizia, Roma, FIVol.

C. Marzotto (1996), «Mini-comunità di persone con handicap fisico-psichico, operatori e volontari: esperienze di vita e modelli a confronto», Politiche Sociali e Servizi, 2.

G.P. Barbetta (1997), « Trasformazioni recenti dei sistemi sanitari e ruolo delle organizzazioni nonprofit», Politiche Sociali e Servizi, 2, pp. 91-128.

G.P. Barbetta, C. Ranci (1997), Terzo settore e nuove politiche sociali: il caso italiano, in G. Rossi (a c. di), Terzo settore, stato e mercato nella trasformazione delle politiche sociali in Europa, cit., pp. 177-214.

I. Colozzi (1997), Il ruolo del terzo settore nei servizi sociali a livello locale, in AIS (a c. di), La transizione italiana degli anni '90, Milano, FrancoAngeli.

L. Freey (1997), «Il ruolo del volontariato nella lotta all'esclusione sociale», Economia pubblica, 5, pp. 5-24.

A. Lovati (1997), Un nuovo ruolo per il volontariato nell'area della giustizia, Padova, Fondazione E. Zancan.

M.L. Mirabile (1997), Il ruolo del terzo settore nelle politiche sanitarie, in I. Colozzi (a c. di), Terzo settore e sviluppo civile: verso una 'regolazione promozionale', cit.

G. Rossi (1997), «Le organizzazioni di volontariato che operano in ambito sanitario», Politiche Sociali e Servizi, 2, pp. 295-314.

U. De Siervo (a c. di) (1998), Regioni e volontariato nei servizi sociosanitari, Milano, Giuffrè.

E. Gastaldi, P. Springhetti (1998), Al di là delle mura. Le ragioni della solidarietà, Roma, FIVol.

M. Anoni, S. Borselli (1999), Partecipare alle politiche sociali, Roma, Carocci.

U. Ascoli, E. Pavolini (1999), «Le organizzazioni di Terzo settore nelle politiche socioassistenziali in Europa: realtà diverse a confronto», Stato e Mercato, 57, pp. 441-476.

M. Bertante (1999), «Il volontariato nei servizi sanitari», Politiche Sociali, 2, pp. 69-75.

R. Caselli, S. Galli, R. Settesoldi (1999), «Qualità dei servizi sanitari, ruolo del volontariato e delle altre forme di privato sociale», Quaderni del Centro Nazionale del volontariato, Lucca.

F. Marsico (1999), Alcune note sulle tendenze attuali del volontariato, nel settore dei servizi alla persona, Padova, Fondazione E. Zancan.

P. Donati (2000), La sanità nonprofit, il ruolo del privato sociale nei servizi sanitari, Rimini, Maggioli.

G. Fiorentini (2000), Pubblico e privato nel nuovo welfare: la regolamentazione delle organizzazioni non lucrative e dei servizi di utilità sociale, Bologna, il Mulino.

R. Frisanco (a c. di) (2000), Non solo carcere. Indagine nazionale sulle organizzazioni di volontariato nell'ambito della giustizia, Roma, FIVol.

G. Nervo (2001), «Il terzo settore favorisce lo sviluppo di un sistema di servizi alla persona universalistico o residuale?», Studi Zancan: Politiche e Servizi alle Persone, 1, pp. 110113.

L. Zen (2001), Il volontariato e il privato sociale nel piano di Zona, Rimini, Maggioli. 
L. Fazzi (2002), Processi di partecipazione attiva dei cittadini nella costruzione di piani sociali, in «Fuori Orario», 33.

G. Bonomi, P. Portioli (2003), Le nuove forme di gestione dei servizi sociali. Alcuni modelli a confronto, in G. Magistrali (a c. di), Il futuro delle politiche sociali in Italia. Prospettive e nodi critici della legge 328/2000, cit., pp. 137-174.

N. Delai (2003), Salute e volontariato. Primo rapporto annuale sull'esperienza sociale del volontariato sanitario e assistenziale, Roma, ILESIS.

AA.VV. (2004), Il ruolo del volontariato in una società multietnica, Cesvot, Firenze, 2.

P. Pierantognetti, L. Bianchi, M. Vario (2004), «Il ruolo del volontariato nelle politiche socioassistenziali», Professioni Infermieristiche, 57, 2, pp. 85-92.

G. D`Angelo, A.M. Gallo, F. Santanera (2005), Volontariato dei diritti: quarant anni di esperienze nei settori della sanità e dell assistenza, Torino, UTET.

G. Rovati (2005), «Le risposte allesclusione sociale: il contributo del sistema non profit», Non profit, 1, pp. 111-150.

I. Colozzi (2006), Paradigma relazionale e innovazioni nelle politiche sociali e di welfare, in P. Donati, I. Colozzi (a c. di), Il paradigma relazionale nelle scienze sociali: le prospettive sociologiche, Bologna, il Mulino, pp. 391-428.

AA.VV. (2007), «Il terzo settore nei sistemi locali di welfare», in Studi Zancan: Politiche e Servizi alle Persone, 4, pp. 58-180.

L. Ferrari (2007), In carcere, scomodi. Cultura e politiche del volontariato, Milano, FrancoAngeli.

M. Accorini (2008), Terzo settore e welfare locale, Roma, Carocci.

D. Barberis (2008), La partecipazione del terzo settore nella fase attuativa del Piano di zona. Spunti e considerazioni dall'esperienza di un Piano di zona, in A. Mosca (a c. di), Il volontariato e il nuovo welfare. Partecipazione, Legge 328/00, programmazione dei servizi in Lombardia, cit., pp. 47-54

D. Cicoletti (2008), «Volontariato e piani di zona in Lombardia», Prospettive Sociali e Sanitarie, $10, \mathrm{pp} .15-18$.

F. Longo (2008), Quale ruolo per il volontariato nella programmazione socio-sanitaria locale?, in A. Mosca (a c. di), Il volontariato e il nuovo welfare. Partecipazione, Legge 328/00, programmazione dei servizi in Lombardia, cit., pp. 55-64.

F. Olivetti Manoukian (2008), Il ruolo del volontariato lombardo nella programmazione sociale di zona, in A. Mosca (a c. di), Il volontariato e il nuovo welfare. Partecipazione, Legge 328/00, programmazione dei servizi in Lombardia, cit., pp. 35-46.

M. Tognetti Bordogna $\left(2008^{3}\right)$, Terzo settore e politiche sociali, in Id., Lineamenti di politica sociale. Cambiamenti normativi e organizzazione dei servizi alla persona, Milano, FrancoAngeli, pp. 200-248.

M. Villani (2008), Tra buone motivazioni e buone prassi: la partita della programmazione zonale, in A. Mosca (a c. di), Il volontariato e il nuovo welfare. Partecipazione, Legge 328/00, programmazione dei servizi in Lombardia, cit., pp. 65-70.

S. Monetini (2009), «Il contributo della società al 'trattamento' dei detenuti, con particolare riguardo al volontariato», Rassegna Penitenziaria e Criminologica, 13, 3, pp. 33-72.

E. Rossi (2009), «Partnership sociali tra pubblico, privato e Terzo Settore: verso lidentificazione di buone pratiche nei servizi alla persona», Aretè, 2, pp. 62-79.

G. Rossi, L. Boccacin (2009), «Il contributo del Terzo settore nel promuovere partnership sociali e buone pratiche nei servizi alla persona: indicazioni di un indagine sociologica», Aretè, 3, pp. 21-34.

S. Licursi, G. Marcello (2010), «Il ruolo del volontariato dove il welfare si fa debole», Auto- 
nomie locali e servizi sociali, XXXIII, 3, pp. 441-458.

\section{L'organizzazione delle associazioni di volontariato}

\subsection{Gestione, promozione e controllo}

G. Rebora (1991), La gestione imprenditoriale e gli aspetti organizzativi delle imprese senza fini di lucro, in C. Borzaga (a c. di), Il terzo sistema. Una nuova dimensione della complessità economica e sociale, cit., pp. 99-104.

M. Ambrosini (1992), «Terzo settore tra efficienza e solidarietà. Un'analisi sul piano organizzativo», Politiche Sociali e Servizi, 2, pp. 7-22.

G. Fiorentini (1992), Organizzazioni non profit e di volontariato. Direzione, marketing e raccolta fondi, Milano, Etas.

M. Ambrosini (a c. di) (1994), L'efficienza della solidarietà, Milano, Vita e Pensiero.

M. Ambrosini (1994), Il terzo settore tra efficienza e solidarietà. Un'analisi sul piano organizzativo, in B. Cattarinussi (a c. di), Altruismo e solidarietà. Riflessioni su prosocialità e volontariati, cit.

D. Cavenago (1996), Dirigere e governare una organizzazione non profit. Economia e management, Padova, Cedam.

I. Colozzi (1996), Problemi di organizzazione, di efficienza ed efficacia delle organizzazioni non profit, G.M. Comolli, R. Garbagnati (a c. di), Volontariato verso il 2000, cit., pp. 8894.

G. Fiorentini (1996), Management efficiente e solidale, in G.M. Comolli, R. Garbagnati (a c. di), Volontariato verso il 2000, cit.

P. Perotto (1996), Quale qualità nel volontariato?, G.M. Comolli, R. Garbagnati (a c. di), Volontariato verso il 2000 , cit.

A. Petrucci (1996), Esigenze del "mercato" e risposte "trasparenti" del non profit, in G.M. Comolli, R. Garbagnati (a c. di), Volontariato verso il 2000, cit.

G. Tomai (1996), Qualità dei servizi erogati, G.M. Comolli, R. Garbagnati (a c. di), Volontariato verso il 2000 , cit.

G. Spreti (1996), Marketing sociale, in G.M. Comolli, R. Garbagnati (a c. di), Volontariato verso il 2000, cit.

A. Carzaniga, C. Fusco Karmann. (1997), «Gestione informatizzata dei volontari: una guida pratica», Prospettive Sociali e Sanitarie, 15-16, pp. 26-29.

A. Fossati, R. Levaggi (1997), «Efficienza e ciclo vitale nelle organizzazioni di volontariato», Economia Pubblica, 1, pp. 111-126.

Agenzia per lo sviluppo del non profit (1998), Il manager del non profit: [le nuove frontiere e le nuove figure professionali dell'imprenditoria sociale], Milano, Sperling \& Kupfer.

G. Ambrosio, F. Bandini. (1998), La gestione del personale nelle organizzazioni non profit, Milano, Etas Libri.

F. Busnelli, C. Gioia (1998), Come si fa a valutare la qualità dei servizi, Roma, FIVol.

F. Busnelli, S. Giuliani (1998), Come si fa a organizzare le risorse umane, Roma, FIVol.

P Milanese (1998), Non-profit marketing e valore sociale, Milano, Egea.

M. Morganti (1998), Nonprofit: produttività e benessere, Milano, FrancoAngeli.

C. Travaglini (1998), «Percorsi di sviluppo e coordinazioni nel Terzo settore: dalle aziende non profit ai gruppi misti del non profit», Non profit, IV, 4, pp. 635-648. 
M. Ambrosini (1999), Identità organizzative e domanda di servizi, in Id. (a c. di), Tra altruismo e professionalità. Terzo settore e cooperazione in Lombardia, cit.

C. Cominelli (1999), La gestione delle risorse umane nelle organizzazioni del terzo settore, in M. Ambrosini (a c. di), Tra altruismo e professionalità. Terzo settore e cooperazione in Lombardia, cit.

D. Mason, V. Melandri (1999), Il management delle organizzazioni non profit, Rimini, Maggioli.

A. Matacena (a c. di) (1999), Aziende non profit. Scenari e strumenti per il Terzo settore, Milano, EGEA.

M. Ambrosini (2000), Le radici dell'altruismo. Basi sociali e peculiarità organizzative dei soggetti del terzo settore, in L. Boccacin, D. Bramanti (a c. di), «Dare, ricevere, fidarsi», Sociologia e Politiche Sociali, 2, pp. 37-64.

G. Ambrosio, R. Bonacina (a c. di) (2000), Manuale per la gestione delle organizzazioni non profit, Milano, Etas.

G.P. Barbetta, C. Schena (a c. di) (2000), Regolazione e controllo sulle organizzazioni nonprofit, Bologna, il Mulino.

F. Bicciato, L. Foschi, F. Di Troia (2000), Valutazione, in L. Fazzi (a c. di), Cultura organizzativa del nonprofit, Milano, FrancoAngeli.

M. Cerri (2000), Il risk management al servizio del non profit, Milano, Egea.

G.M. Colombo (2000), «Complessità organizzativa nel volontariato», Prospettive Sociali e Sanitarie, XIX, pp. 14-16.

L. Fazzi (2000), Decisioni, in Id. (a c. di), Cultura organizzativa del nonprofit, cit.

M. Crescenzi (a c. di) (2002), Manager e management non Profit, Roma, ASVI Sviluppo Non Profit Edizioni.

V. Melandri (2002), Fund Raising e accountability, in S. Zamagni (a c. di), Il non profit italiano al bivio, Milano, Egea, pp. 153-195.

P. Atzei (2003), La gestione dei gruppi nel terzo settore. Guida al cooperative learning, Roma, Carocci.

D. Converso, D. Piccardo (2003), Il profitto dell'empowerment. Formazione e sviluppo organizzativo nelle imprese non profit. Milano, Raffaello Cortina Editore.

E. De Palma (2003), «La valutazione dell'operato dei volontari: strumento strategico per lo sviluppo delle risorse umane», Terzo Settore, 9, pp. 37-44.

E. De Palma (2003), «Professionisti e Volontari: superare i timori per integrare le risorse», Non profit, 3, pp. 589-606.

E. De Palma (2003), «Come valorizzare i volontari», Rivista del Volontariato, 10, 11, pp. 4144.

G. Lazzarini (2003), Universi solidali: il terzo settore tra gratuità e organizzazione efficiente, Torino, Edizioni Gruppo Abele.

F. Lertora (a c. di) (2003), Il gruppo di volontariato istruzioni per l'uso. Manuale per la conduzione di gruppo, Genova, Celivo.

B. Sibilio Parri (2003), «Il sistema di controllo nelle aziende non profit», Non profit, IX, 2, pp. 151-180.

L. Tronca (2003), «Dalla teoria della governance alla social governance: un ipotetico stile autoregolativo per le organizzazioni di Terzo settore», Impresa Sociale, 71-72, pp. 65-84.

M. Catalano (2004), Le buone prassi di bilancio sociale nel volontariato, n. 21, Firenze, Cesvot.

M.P. Mostarda (2004), «Il volontariato tra scelte individuali e processi organizzativi», Dirigenti Scuola, 25, 2, pp. 46 ss. 
F. Piancastelli (2004), Caratteristiche organizzative e orientamenti culturali all'interno del Privato sociale, in P. Donati, I. Colozzi (a c. di), Il terzo settore in Italia: culture e pratiche, cit., pp. 257-280.

F. Busnelli (2005), La gestione delle persone nelle organizzazioni non profit, in A. Hinna (a c. di), Gestire e organizzare nel Terzo settore, Roma, Carocci, pp. 221-242.

Eu-tròpia (a c. di) (2005), Volontariato: professione benessere. Il benessere organizzativo nel non profit, Milano, FrancoAngeli.

AA.VV. (2006), Il miglioramento della qualità nelle Associazioni di Volontariato, Milano, FrancoAngeli.

L. Boccacin (2006), Le differenti forme organizzative del volontariato in Italia, in Id., G. Rossi, Le identità del volontariato in Italia. Orientamenti valoriali e stili di intervento a confronto, Milano, Vita e Pensiero, pp. 38-40.

L. Bagnoli (2007), Il bilancio sociale delle organizzazioni di volontariato, n. 34, Firenze, Cesvot.

S. Ragghianti, R. Settesoldi (2007), Le responsabilità degli organi amministrativi delle associazioni di volontariato, n. 35, Firenze, Cesvot.

R. Cerabolini (2008), «Il volontariato tra motivazione e organizzazione», Prospettive Sociali e Sanitarie, 4, pp. 15-17.

I. Colozzi (2008), Introduzione. Perché una ricerca sui leader del Terzo settore, in Id., R. Prandini (a c. di), I leader del terzo settore. Percorsi biografici, culture e stili di leadership, cit.

I. Colozzi (2008), Conclusioni. Un ritratto abbozzato della leadership del Terzo settore in controluce della società italiana, in Id., R. Prandini (a c. di), I leader del terzo settore. Percorsi biografici, culture e stili di leadership, cit.

I. Colozzi (2008), «Leader del Terzo settore e capacità sociale», Aretè, 2, pp. 39-49.

R. Prandini (2008), Percorsi biografici, capitale sociale e orientamenti valoriali dei leader delle organizzazioni di volontariato, in I. Colozzi, R. Prandini (a c. di), I leader del terzo settore. Percorsi biografici, culture e stili di leadership, cit.

A. Bilotti, L. Nasi, P. Tola, A. Volterrani (2009), La valutazione di impatto sociale dei progetti del volontariato toscano, n. 43, Firenze, Cesvot.

Fondazione E. Zancan (2009), «Responsabilità professionali e direzionali nel terzo settore», Studi Zancan: Politiche e Servizi alle Persone, 2, pp. 71-170.

S. Zicari, S. Martello (2009), «L'accoglienza del volontario: modalità operative ed obiettivi», Terzo Settore, 4, pp. 48-51.

S. Macchioni (a c. di) (2010), L'infrastruttura del volontariato in Italia, Roma, CSVnet.

M. Morganti $\left(2010^{2}\right)$, Non profit: produttività e benessere. Come coniugare efficienza e solidarietà nelle organizzazioni del terzo settore, Milano, FrancoAngeli.

L. Simonelli (2010), «Quale tutela per volontari e collaboratori gratuiti?», Enti Non Profit, 12,6 , pp. 20-24.

\subsection{La formazione}

G. Bocca (1993), «La formazione del volontario: aspetti pedagogici ed educativi», Vita $e$ Pensiero, LXXVI, pp. 779-787.

D. Bramanti (1993), «La formazione, uno strumento per operare. Analisi del ruolo della formazione nell'azione volontaria: tra desiderio di appartenenza e richiesta di professionali- 
tà», La Ricerca Sociale, XLIX, pp. 242-261, poi in I. Colozzi (a c. di), Terzo settore e nuove politiche sociali in Italia e in Europa, cit.

D. Bramanti (1994), «Il ruolo della formazione nelle organizzazioni di volontariato», Politiche Sociali e Servizi, 1, pp. 39-60.

L. Prenna (1995), La solidarietà voluta. formazione di base del volontariato, Roma, Città Nuova.

D. Bellamio (1996), Formazione per la qualità nel volontariato, in G.M. Comolli, R. Garbagnati (a c. di), Volontariato verso il 2000, cit.

L. Beretta, V. Melandri (1996), «Aspetti problematici della formazione nelle organizzazioni non profit», Non profit, 2, pp. 163-175.

D. Bramanti (1996), Rapporto qualità-formazione, in G.M. Comolli, R. Garbagnati (a c. di), Volontariato verso il 2000, cit., pp. 129-135.

F. Busnelli (1996), La formazione del volontariato, in V. Gallina, M. Lichtner (a c. di), L'educazione in età adulta, Milano, FrancoAngeli, pp. 117-123.

L. Gangeri (1996), Sapere, saper fare, saper essere: tre livelli conoscitivi nella formazione del volontariato, in G.M. Comolli, R. Garbagnati (a c. di), Volontariato verso il 2000, cit.

G. Lombardi (1996), Integrazione fra volontariato e strutture pubbliche: gli effetti della formazione, in G.M. Comolli, R. Garbagnati (a c. di), Volontariato verso il 2000, cit.

V. Melandri (1996), «Il problema formativo nel settore non profit», Non profit, 3, pp. 249272.

V. Melandri (1997), «I bisogni formativi dei managers non profit italiani: un'indagine sul campo», Non profit, 4, pp. 505-539.

A. Cevolini (2002), Mezzi, risorse e cultura della rendicontabilità nelle organizzazioni di privato sociale, in P. Donati, I. Colozzi I. (a c. di), La cultura civile in Italia, cit., 133162.

E. De Palma (2002), I volontari una risorsa da valorizzare, Troina, Città Aperta.

G. Pellegrini (a c. di) (2005), Azione volontaria e sfide per la formazione, Roma, Carocci.

S. Rizza (2005), «Professioni sociali e formazione», Rassegna di Servizio Sociale, IVL, 1, pp. 5-7.

I. Sapienza (2005), «La formazione dei volontari», Terzo Settore, 1, pp. 13-20.

F. Marcon (2006), «Non profit: investire in formazione», Terzo Settore, 12, pp. 48-50.

S. Balbi, G. Boccuzzo, M.G. Grassia (a c. di) (2008), Profili formativi e bisogni di competenze nel terzo settore, CLEUP, Padova.

V. Melandri (2010), «Valorizzare i volontari e il personale retribuito», Terzo Settore, 6, pp. 44-48.

G. Sordelli (a c. di) (2010), Volontariato e formazione a distanza, n. 49, Firenze, Cesvot.

\subsection{La comunicazione}

R. Franzoni (a c. di) (1993), Volontariato e informazione, Roma, FIVol.

A. Volterrani (1994), Volontariato e mass media. Un rapporto difficile, in B. Cattarinussi (a c. di), Altruismo e solidarietà. Riflessioni su prosocialità e volontariati, cit.

C. Giobbio (1994), La comunicazione integrata nel settore non profit, in R. Fiocca (a c. di), $\mathrm{La}$

comunicazione integrata nelle aziende, Milano, EGEA. pp. 315-347.

S. Ragghianti (1998), «Volontariato, intervento di rete, nuove Tecnologie», Volontariato Oggi, 3. 
V. Albanesi don, (1999) Marginalità della comunicazione del volontariato: riflessioni e proposte, in C. Tisselli (a c. di), L'informazione del volontariato. Linguaggio, nuove tecnologie e forme di tutela, Milano, FrancoAngeli.

F. Franzoni (1999), Comunicare la solidarietà: il ruolo del volontariato nel welfare, in C. Tisselli (a c. di), L'informazione del volontariato. Linguaggio, nuove tecnologie e forme di tutela, cit.

N. Kanzian (1999), Comunicare nel non profit. Comunicazione e logica per la formazione, Trieste, Battello.

M.E. Martini (1999), Sviluppo della comunicazione di massa e crescita di una responsabilità solidale, in C. Tisselli (a c. di), L'informazione del volontariato. Linguaggio, nuove tecnologie e forme di tutela, cit.

E. Mori (1999), La soggettività comunicativa del volontariato, in C. Tisselli (a c. di), L'informazione del volontariato. Linguaggio, nuove tecnologie e forme di tutela, cit.

A. Volterrani (1999), La comunicazione per il volontariato, n. 6, Firenze, Cesvot.

A. Volterrani (1999), Telematica e comunicazione, in C. Tisselli (a c. di), L'informazione del volontariato. Linguaggio, nuove tecnologie e forme di tutela, cit.

R. Frisanco, S. Trasatti, A. Volterrani (a c. di) (2000), La voce del volontariato. Indagine nazionale su organizzazioni di volontariato e comunicazione, Roma, FIVol.

S. Martelli (2006), Comunicare il capitale sociale: le attività e le iniziative delle Organizzazioni di Terzo settore a Palermo, in P. Donati, I. Colozzi (a c. di), Terzo settore e valorizzazione del capitale sociale in Italia: luoghi e attori, cit., pp. 247-278.

F. Pira (2005), Come comunicare il sociale. Strumenti, buone pratiche e nuove professioni, Milano, FrancoAngeli.

G. Gili (2008), Il Terzo Settore e la sfida della comunicazione, in I. Barholini (a c. di), Capitale sociale, reti comunicative e culture di partecipazione, cit., pp. 59-74.

S. Martelli (2008), La comunicazione come risorsa del Terzo Settore per la società civile del Sud, in I. Barholini (a c. di), Capitale sociale, reti comunicative e culture di partecipazione, cit., pp. 105-124.

R. Sobrero (2008), «La comunicazione, strumento di sviluppo per il terzo settore», Aretè, 2, pp. 85-100.

P. Springhetti (2008), Solidarietà indifesa. L'informazione nel sociale, Bologna, EMI della Coop. SERMIS.

\subsection{Il networking}

M. Ambrosini, A. De Bernardis (1999), Assetti organizzativi e rapporti con i contesti di origine, in M. Ambrosini (a c. di), Tra altruismo e professionalità. Terzo settore e cooperazione in Lombardia, cit.

G. Costa (2000), «La costruzione della partnership», Prospettive Sociali e Sanitarie, 13, pp. $5-8$.

C. Guccinelli, R. Podestà (2000), Dare credito all'economia sociale. Strumenti del credito per soggetti non profit, n. 12, Firenze, Cesvot.

A. Andreotti, P. Barbieri (2003), «Reti e capitale sociale», Inchiesta, XXXIII, 139, pp. 1-4.

O. Osio (2005), «Un progetto di rete per la promozione del volontariato», Lavoro Sociale, V, 2, pp. 245-252.

A. Salvini, D. Cordaz (2007), Coesione strutturale e capitale sociale. Analisi di rete del volontariato locale, paper presentato al Convegno AIS Metodologia: Esplorare la coesione 
sociale. Teorie, ipotesi, modelli, tecniche di analisi dei dati, Trento 28-30.6.2007.

L. Boccacin (2010), «Le forme di partnership sociale: il contributo della riflessione sociologica alla loro comprensione», Sociologia e Politiche Sociali, XIII, 3, pp. 9-27.

D. Bramanti (2010), «Le partnership: costruire relazioni fiduciarie e promuovere buone pratiche», Sociologia e Politiche Sociali, XIII, 3, pp. 45-67.

\subsection{Il fundraising e il people raising}

G. Cacopardo (1996), Obiettivi e strategie di 'fundraising', in G.M. Comolli, R. Garbagnati (a c. di), Volontariato verso il 2000, cit.

S.A. Castegnaro (1996), Sponsorizzazioni, in G.M. Comolli, R. Garbagnati (a c. di), Volontariato verso il 2000 , cit.

F. Manfredi (2000), Risorse, in L. Fazzi (a c. di), Cultura organizzativa del nonprofit, cit.

M. Griffino (2002), «Il finanziamento delle organizzazioni di volontariato», Terzo Settore, 5.

J.S. Ellis, V. Melandri (2003), People Raising. Manuale pratico per la ricerca di volontari, Roma, Carocci.

R. Bemi (2004), Le opportunità "finanziare e reali" per le associazioni di volontariato toscane. Le principali fonti di finanziamento locali, zonale, provinciale, regionale e nazionale, n. 23, Firenze, Cesvot.

E. De Palma (2004), «Dare continuità all'azione volontaria: come incentivare la partecipazione dei volontari», Relazioni Solidali, 1.

S. Lemmetti (2004), Raccolta fondi per le Associazioni di Volontariato. Criteri ed opportunità, n. 22, Firenze, Cesvot.

S. Lemmetti (2006), Buone prassi di fund raising nel volontariato toscano, n. 33, Firenze, Cesvot.

R. Bemi (2008), Contributi e finanziamenti per le associazioni di volontariato. Guida prati$c a$, n. 38, Firenze, Cesvot.

S. Martello, S. Zicari (2009), «Il reclutamento dei volontari nel Terzo Settore», Terzo Settore, 9, pp. 43-46.

R. Bemi (2010), Contributi e finanziamenti per le associazioni di volontariato. Aggiornamento 2009, n. 48, Firenze, Cesvot.

G. Solfrini (2010), «Come reclutare il volontario ideale», Terzo Settore, 4, pp. 36-37.

\section{I volontari: profili socio-demografici, motivazioni e orientamenti valoriali}

V. Cremoncini (1991), «Anziani e volontariato», Orientamenti, 1.

V. Cremoncini, T. Vecchiato (a c. di) (1991), Ruolo del volontariato nella assistenza agli anziani, Padova, Fondazione E. Zancan.

G. Milanesi (1992), «Giovani e volontariato», Rivista del Volontariato, 4, pp. 16-19.

R. Franzoni (a c. di) (1993), Volontariato e giovani. Forme e motivazioni diverse per una generazione in cerca di valori, Roma, FIVol.

G. Milanesi (a c. di) (1993), «Volontariati in Europa», Quaderni di Volontariato, 3, Roma, FIVol.

G. Sarpellon, (1993), Solidarietà, altruismo, interesse, in P. Donati, G.B. Sgritta (a c. di), Cittadinanza e nuove politiche sociali, cit., pp. 234 ss.

G. Badolato, M.C. Rizzi (1994), «Alla ricerca dell'identità e dell'appartenenza. Significati dell'esperienza di volontariato giovanile», Animazione Sociale, XXIV, 12, pp. 22-28. 
G. Rossi (1996), Motivazioni e orientamenti pro-sociali, in G.M. Comolli, R. Garbagnati (a c. di), Volontariato verso il 2000, cit.

C. Degiacomi, M.R. Gilliavod (a c. di) (1997), Volontariato, Volontariati. Guida Ragionata. Aggiornamento Annuale, Torino, Edizioni Radionotizie.

R. Frisanco (1998), «Giovani volontari», Rivista del Volontariato, 7-8, pp. 41-43.

P. Springhetti (a c. di) (1998), «Volontari nella transizione», Rivista del Volontariato, 2, pp. 29-35.

IREF-ACLI (1999), La società civile in Italia. Indagine sulla adesione associativa, l'impegno volontario e le donazioni della popolazione italiana, Roma, Edizioni del Lavoro.

U. Ascoli (1999), «I volontariati», Prospettive Sociali e Sanitarie, 11, pp. 3-5.

C. Arcidiacono, P. Menna, F. Procentese (2004), Il volontariato e forme di associazionismo: rappresentazioni e identità in una realtà meridionale, in $\mathrm{C}$. Arcidiacono (a c. di), Volontariato e legami collettivi. Bisogni di comunità e relazione reciproca, cit., pp. 107-124.

R. Oldini (2000), «Giovani e volontariato: una svolta generativa», Ambrosius, 6.

E. D’Orazio (2001), Elogio e critica del volontariato: fare volontariato nel 21. secolo in Italia e in Europa: gli anziani protagonisti. Roma, EDUP.

L. Solari, Appartenenze (2000), in L. Fazzi (a c. di), Cultura organizzativa del nonprofit, cit.

C. Capanna, P. Steca, A. Imbimbo (2002), «Una scala per la misura della motivazione al volontariato», Rassegna di Psicologia, XIX, 1, pp. 73-90.

I. Ivaldi, S. Polidori (a c. di) (2002), Giovani, volontariato e servizio civile: situazione e prospettive. Un'indagine esplorativa, Roma, Osservatorio Nazionale per il volontariato.

C. Barbaranelli, G.V. Caprara, C. Capanna, A. Imbimbo (2003), «Le ragioni del volontariato: un contributo empirico», «Giornale italiano di psicologia», XXX, 2, pp. 369-388.

L. Boccacin (2003), Giovani e volontariato organizzato: trend emergenti dalla ricerca sociologica, in E. Marta, E. Scabini (a c. di), Giovani volontari: impegnarsi, crescere e far crescere, Firenze, Giunti, pp. 144-172.

M. Ambrosini (a c. di) (2004), Per gli altri e per sé. Motivazioni e percorsi del volontariato giovanile, Milano, FrancoAngeli.

L. Boccacin (2004), Itinerari alla prosocialità dei giovani adulti volontari: indicazioni da una indagine empirica, in Id., G. Rossi (a c. di), «Stili partecipativi emergenti nel volontariato giovanile», Sociologia e politiche sociali, VII, 2, pp. 43-58.

A. Fedi, K. Greganti, S. Tartaglia (2004), Quale partecipazione, quale identità?, in C. Arcidiacono (a c. di), Volontariato e legami collettivi. Bisogni di comunità e relazione reciproca, cit., pp. 56-75.

R. Frisanco (2004), Volontariato e giovani nel nuovo secolo, in L. Boccacin, G. Rossi (a c. di), «Stili partecipativi emergenti nel volontariato giovanile», Sociologia e politiche sociali, VII, 2, pp. 93-114.

G. Rossi (2004), La trasmissione della prosocialità tra le generazioni: il caso delle famiglie dei giovani-adulti volontari, in L. Boccacin, Id. (a c. di), «Stili partecipativi emergenti nel volontariato giovanile», Sociologia e politiche sociali, VII, 2, pp. 59-76.

Marta Elena (2004), Matrice familiare e storia intergenerazionale nella scelta di impegno nel volontariato dei giovani, in L. Boccacin, G. Rossi (a c. di), «Stili partecipativi emergenti nel volontariato giovanile», Sociologia e politiche sociali, VII, 2, pp. 77-92.

M. Ambrosini (2005), «Giovani e volontariato: impegno per altri e crescita personale», $A g$ giornamenti Sociali, LXVI, 3, pp. 183-194.

V. Pieroni (2005), «Volontari 'perché'. Dalla pedagogia dell'alterità paradigmi e paradossi», Orientamenti Pedagogici, 52, 1, pp. 9-24.

E. Marta, C. Guglielmetti, M. Pozzi (2006), «Volunteerism during young adulthood: An ital- 
ian investigation into motivational patterns», Voluntas, 17, 3, pp. 221-232.

G. Pirozzi (2006), «Giovani per quale volontariato? Interazioni tra giovani e mondo del volontariato per una democrazia partecipata», Animazione Sociale, XXXVI, 11, pp. 76-84.

L. Guizzardi (2007), «Capitale sociale e volontariato dei giovani adulti: una ricerca», Sociologia e Politiche Sociali, 1, pp. 143-159.

E. Marta, M. Pozzi (2007), Psicologia del volontariato, Roma, Carocci.

P. Previtali (2007), «La motivazione del volontario: alcune evidenze empiriche», Non profit, 2, pp. 591-603.

E. Spedicato Iengo (2007), «Il volontariato: da prassi consolatoria a impegno progettuale», Oggi Domani Anziani, 4, pp. 79-96.

R. Cerabolini (2008), «Il volontariato tra motivazione e organizzazione», Prospettive Sociali e Sanitarie, 4, pp. 15-17.

C. Grano, F. Lucidi, A. Zelli, C. Violani (2008), «Motives and Determinants of Volunteering in Older Adults: an Integrated Model», International Journal of Aging \& Human Development, 67, 4, pp. 305-326.

G. Sensi (2008), «Giovani, volontariato, democrazia», Volontariato Oggi, 24, 2, pp. 12-13.

U. Ascoli (2009), «Introduzione. I “volontariati” oggi in Italia», Impresa Sociale, XIX, 4, pp. 11-18.

G. Degli Antoni (2009), Motivations to volunteer and social capital: the role of intrinsic motivations in promoting networks of cooperative relations, Econometica, WP. n. 6, Milano-Trento-Siena-Bologna-Verona, Centro interuniversitario per l'etica economica e la responsabilità sociale d'impresa.

R. Frisanco (2009), Identità del volontariato: confronto intergenerazionale, Oggi Domani Anziani, 4, pp. 59-73.

R. Di Gioia, L. Giacomello, P.P. Inserra, S. Rotondi (2010), Quando i giovani partecipano. Prima indagine nazionale sulla presenza giovanile nell'associazionismo, nel volontariato e nelle aggregazioni informali, Roma, Sviluppo locale Edizioni.

D. Maran, G. Soro (2010), «The influence of organizational culture in women participation and inclusion in voluntary organizations in Italy», Voluntas, XXI, 4, pp. 481-496.

I. Psaroudakis (2010), Profili del volontario. Nuove direzioni della gratuità, in M.A. Toscano (a c. di), ZoonPolitikon 2010. Politiche sociali e partecipazione, cit., pp. 111-124.

F. Romano, O.M. Todaro (2010), «Le ragioni del volontariato: dare o ricevere?», in Psicologia di Comunità, 1, pp. 139-144.

A. Salvini (a c. di) (2010), Profili dei volontari in Toscana, Pisa-Firenze, Dipartimento di Scienze Politiche e Sociali, Università di Pisa-Cesvot.

\section{La rappresentazione del volontariato}

R. Bonacina (a c. di) (1998), «Il Volontariato in pagina. La rappresentazione del volontariato sulla stampa quotidiana italiana», Vita, 50, suppl.

A. Balzanelli (1999), Storie del volontariato: come far passare le buone notizie, in C. Tisselli (a c. di), L'informazione del volontariato. Linguaggio, nuove tecnologie e forme di tutela, cit.

A. Fedi, S. Gattino (2004), La rappresentazione della solidarietà e del volontariato: alcune note teoriche ed un esempio di ricerca qualitativa, in C. Arcidiacono (a c. di), Volontariato e legami collettivi. Bisogni di comunità e relazione reciproca, cit., pp. 23-43.

M. Forestieri, C. Magro (2004), Il volontariato messinese attraverso la stampa. Torino, EGA.

A. Volterrani (a c. di) (2006), Raccontare il volontariato, n. 29, Firenze, Cesvot. 
C. Sorrentino (a c. di) (2007), Ultime notizie! La rappresentazione del volontariato nella stampa toscana, n. 37, Firenze, Cesvot.

\section{Le rappresentanze: Fondazione italiana, Centro nazionale, Mo.vi., Csv, etc.}

E. Carretta (1996), Movi (Movimento volontariato italiano), in G.M. Comolli, R. Garbagnati (a c. di), Volontariato verso il 2000, cit.

G. Macaluso (1996), Università del Volontariato, in G.M. Comolli, R. Garbagnati (a c. di), Volontariato verso il 2000, cit.

M.E. Martini (1996), Centro Nazionale per il volontariato di Lucca, in G.M. Comolli, R. Garbagnati (a c. di), Volontariato verso il 2000, cit.

G. Memo (1997), «I Centri di Servizio per il Volontariato», Prospettive Sociali e Sanitarie, 10 , pp. $1-5$

A. Pancaldi (a c. di) (1998), «Centri di servizio per il volontariato», Quaderni del Cdh, 13, Bologna, CDH Bologna.

F. Merlini (1999), «I Centri di Servizio per il Volontariato: percorsi costitutivi, caratteri e problematiche», Politiche Sociali e Servizi, 2, pp. 313-329.

L. Tavazza (1999), «La Fondazione Italiana per il Volontariato», Aggiornamenti sociali, L, 6, pp. 459-471.

M. Ampollini (2000), «Il volontariato e i Centri di Servizio», Aggiornamenti sociali, LI, 1, pp. 744-755.

Collegamento nazionale centri di servizio per il volontariato \& Cesiav (2000), I Centri di servizio per il volontariato in Italia. Presenza, struttura, servizi. Rapporto 2000, Milano.

G.P. Barbetta (2001), «I CSV: un punto di vista controcorrente», Prospettive Sociali e Sanitarie, 13, pp. 9-10.

Collegamento Nazionale dei Centri di Servizio per il Volontariato (2001), I centri di servizio per il volontariato in Italia, Milano.

M. Granelli (2001), «I Csv, strumento significativo per lo sviluppo del volontariato», Prospettive Sociali e Sanitarie, 13, pp. 8-9.

M.E. Martini (2001), «La concertazione nel funzionamento dei CSV», Prospettive Sociali e Sanitarie, 13, pp. 10-11.

E. Rossi (2001), «I CSV possono sostenere progetti di intervento», Prospettive Sociali e Sanitarie, 13 , pp. 6-7.

M. Serofilli (2001), Alla ricerca della missione dei Csv: che cosa significa "sostenere e qualificare l'attività di volontariato"?, in Id. (a c. di), Promuovere la progettualità del volontariato. Riflessioni sulla progettazione sociale dei Centri di Servizio per il Volontariato in Emilia-Romagna, FrancoAngeli, Milano.

M. Serofilli (2001), Osservazioni, puntualizzazioni e orientamenti per il futuro, in Id. (a c. di), Promuovere la progettualità del volontariato. Riflessioni sulla progettazione sociale dei Centri di Servizio per il Volontariato in Emilia-Romagna, FrancoAngeli, Milano.

CESIAV (a c. di) (2004), I centri di servizio per il volontariato in Italia: presenza, struttura e servizi, Roma, Coordinamento Nazionale dei Centri di Servizio per il Volontariato.

M. Forestieri (2005), «I Centri di servizio per il volontariato tra rendicontazione e valutazione», Prospettive Sociali e Sanitarie, 10-11, pp. 25-28.

CSVnet (2010), Report 2008 - 2009. Resoconto delle attività 2008-2009 dei Centri di Servizio per il Volontariato, Roma. 